\title{
ARTICLE OPEN Mucosal T follicular helper cells in SIV-infected rhesus macaques: contributing role of IL-27
}

\author{
Félicien Moukambi ${ }^{1}$, Henintsoa Rabezanahary ${ }^{1}$, Yasmina Fortier ${ }^{2}$, Vasco Rodrigues ${ }^{2}$, Julien Clain ${ }^{1}$, Ghita Benmadid-Laktout ${ }^{1}$, \\ Ouafa Zghidi-Abouzid ${ }^{1}$, Calayselvy Soundaramourty ${ }^{2}$, Mireille Laforge ${ }^{2}$ and Jérôme Estaquier ${ }^{1,2}$
}

\begin{abstract}
Mesenteric lymph nodes (MLNs), that drain the large and small intestine, are critical sites for the induction of oral tolerance. Although depletion of CD4 T cells in the intestinal lamina propria is a hallmark of HIV infection, CD4 T cell dynamics in MLNs is less known due to the lack of accessibility to these LNs. We demonstrate the early loss of memory CD4 T cells, including T follicular helper cells (Tfh) and a remodeling of MLN architecture in SIV-infected rhesus macaques (RMs). Along with the loss of Tfh cells, we observe the loss of memory B cells and of germinal center B cells. Tfh cells display a Th1 profile with increased levels of the transcription factors that negatively impact on Tfh differentiation and of Stat5 phosphorylation. MLNs of SIV-infected RMs display lower mRNA transcripts encoding for IL-12, IL-23, and IL-35, whereas those coding for IL-27 are not impaired in MLNs. In vitro, IL-27 negatively impacts on Th cells and recapitulates the profile observed in SIV-infected RMs. Therefore, early defects of memory CD4 $\mathrm{T}$ cells, as well of Tfh cells in MLNs, which play a central role in regulating the mucosal immune response, may have major implications for Aids.
\end{abstract}

Mucosal Immunology (2019) 12:1038-1054; https://doi.org/10.1038/s41385-019-0174-0

\section{INTRODUCTION}

Depletion of peripheral blood CD4 T cells and viral load are key parameters in the follow-up of the disease progression to AIDS. It has been reported that chronic immune T-cell activation and apoptosis correlate with disease progression in human immunodeficiency virus (HIV)-infected humans ${ }^{1-4}$, and in the primate model of pathogenic lentiviral infections. ${ }^{5-12}$ Furthermore, several studies have highlighted the profound loss of CD4 T cells in the gut lamina propria linked with microbial translocation. ${ }^{13,14}$ Mesenteric lymph nodes (MLNs) constitute a specialized lymphoid organ, essential in the genesis of the intestinal immune response, as well as draining the gut-associated lymphoid tissue (GALT). MLNs that are disseminated along the colon and at the base of the thoracic lymphatic duct form the cisterna chyli. In response to the penetration of infectious agents through the intestinal barrier, antigen-presenting cells (APCs) carry microbial antigens via the afferent lymph to MLNs. Therefore, resident T cells in MLNs are kept in a state of "immunological tolerance", 15 through the action of immunosuppressive environmental factors such as TGF- $\beta$ and IDO1, contributing to the absence of effector CD8 T cells during the simian immunodeficiency virus (SIV) infection. ${ }^{16}$ However, our knowledge of the CD4 T-cell dynamics in MLNs that drain the GALT is limited, due to the non-accessibility of these LNs in HIVinfected individuals.

$T$ follicular helper (Tfh) cells control germinal center (GC) development and are essential to sustaining antiviral antibody production. ${ }^{17,18}$ Tfh cells, which are rare in the blood, produce IL$21^{19}$, and are specialized providers of $T$ cell help to $B$ cells. ${ }^{20-23} \mathrm{Tfh}$ cells selectively express programmed death molecule 1 (PD-1) and CXC chemokine receptor 5 (CXCR5, originally named MDR15/ BLR1). ${ }^{24-26}$ Thus, Th cells are recruited to lymphoid organs via the follicle-associated chemokine CXCL13/BCA-1 (B cell-attracting chemokine 1). ${ }^{27,28}$ Other groups and ours have reported a defect in Tfh cells during HIV and SIV infections. ${ }^{29-34}$ Thus, HIV-infected individuals with less than $200 \mathrm{CD} 4 \mathrm{~T}$-cells $/ \mathrm{mm}^{3}$ show a deficiency in IL-21-secreting CD4 T cells. ${ }^{35}$ Furthermore, higher numbers of Tfh in the peripheral LNs of nonprogressor, compared to progressor SIV-infected rhesus macaques (RMs) have been reported, ${ }^{36,37}$ and splenic Tfh cells are depleted early after SIV infection. ${ }^{33}$ Consistent with such a defect of Tfh cells, impairment in B-cell function occurring early after HIV infection was reported previously. ${ }^{38-40}$ Hence, rapid progression to AIDS is commonly associated with impaired anti-SIV antibodies in $\mathrm{RMs}^{16}$ and depletion of B cells leads to death in SIV-infected Pigtail macaque. ${ }^{41}$ The frequency and quality of Env-specific Th cells correlates with the genesis of Env-specific B cells and neutralization. ${ }^{37}$ Cubas et al. ${ }^{31}$ have proposed that excessive and persistent triggering of PD-1 on LN Tfh cells may affect their ability to provide adequate B-cell help. ${ }^{32}$ Thus, Tfh cells are of crucial importance in maintaining efficient B-cell immunity in lymphoid tissues. However, the dynamics of Th cells and their relationship with B-cell dynamics in MLNs remain poorly addressed, particularly during the acute phase of infection.

Several transcriptional factors (TFs), including activator and repressor factors, have been reported to play a major role in regulating Tfh cell differentiation. ${ }^{42-46}$ Bcl6 promotes Tfh differentiation, at least in part by suppressing the expression of Tbet (a Th1 TF) ${ }^{45}$ RORyt (Th17), ${ }^{42}$ GATA3 (Th2), ${ }^{46}$ and Blimp-1. 25,47,48 The TFs, C-Maf and TCF1, have also been reported to be involved in the differentiation and/or function of Tfh cells. ${ }^{43,44,49-52}$ On the other hand, the Krüppel-like factor 2 (KLF2) and Foxo1 restrain Tfh cell

\footnotetext{
${ }^{1}$ Centre de Recherche du CHU de Québec, Université Laval, Québec G1V 4G2 QC, Canada and ${ }^{2}$ CNRS FR3636, Université Paris Descartes, 75006 Paris, France
} Correspondence: Jérôme Estaquier (estaquier@yahoo.fr)

Received: 1 August 2018 Revised: 4 April 2019 Accepted: 17 April 2019

Published online: 21 May 2019 
differentiation by inhibiting CXCR5 and Bcl6 expression, ${ }^{53,54}$ and regulating the expression of CD62L. ${ }^{55,56}$

Environmental factors, such as cytokines, are critical in regulating Tfh cell differentiation. Whereas IL- 6 and IL-21 are essential to induce the expression of $\mathrm{Bcl} 6,{ }^{17,57,58} \mathrm{IL}-2$, and IL-7 blocked Tfh cell differentiation by inducing STAT5 and T-bet signaling in activated CD4 T cells, or by repressing Bcl6 and CXCR5 expressions. ${ }^{43,44,59} \mathrm{IL}-12$, involved in the generation of Th1 cells, has been reported to induce Tfh differentiation from human naive cells in the presence of IL- 6 and IL-21. ${ }^{60,61}$ Furthermore, it has also been shown that individuals deficient for the $\beta$ chain of the IL-12 receptor have reduced GC responses and Tfh cells. ${ }^{62}$ IL-23, which shares with IL-12 the IL-12R 1 chain ${ }^{63}$ induces the expression of CXCR5 and ICOS on human naive CD4 T cells. ${ }^{64}$ An additional member of the IL-12 cytokine family, IL-27, has also been involved in boosting Tfh differentiation in mice ${ }^{65-67}$ but not in human cells ${ }^{61}$ suggesting differences between humans and mice regarding the role of IL-27 in the development of Tfh cells. In the context of an HIV infection, a positive correlation was reported between the levels of IL-27 in the plasma and of proviral DNA in peripheral blood mononuclear cells, ${ }^{68}$ whereas it has been shown that IL-27 inhibits the in vitro HIV infection. ${ }^{69,70}$ Furthermore, other groups as well as ours have previously reported a defect in the expression of mRNAs encoding for p40 and p35 of IL-12. ${ }^{2,71}$ However, the impact of IL-27 on the differentiation of Tfh cells derived from MLNs, in the context of SIV infection, is unknown.

Herein, we have performed the analysis of MLN Tfh cells in SIVinfected RMs in relationship with B-cell dynamics and differentiation. Our results indicate a Th1-like Tfh cell profile and an early loss after an infection associated with the loss of memory B cells. Our results also highlight a dysregulation in the TF network related to the increased levels of inhibitory TFs in Tfh cells, and demonstrates, for the first time to our knowledge, the negative impact of IL-27 on Tfh cell differentiation from MLN. Because MLNs are crucial in maintaining commensal microbiota under control, therefore, the loss of memory CD4 T cells, including Tfh cells, may contribute to the absence of gut immunity favouring microbial translocation during an HIV infection.

\section{RESULTS}

T- and B-cell dynamics in MLNs of SIV-infected RMs

Our knowledge of T- and B-cell dynamics in MLNs is limited, during the acute phase, due to the limited accessibility to these LNs in HIV-infected individuals. We analyzed their dynamics in uninfected and SIV-infected RMs. The percentages, as well the numbers of CD4, CD8, and B cells in MLNs that drain the upper (ileocolic, $\Delta$ ) and the lower (colic, O) part of the intestine at different time points postinfection, were assessed (Fig. 1). A significant decrease in the percentage of $\mathrm{CD}^{+} \mathrm{T}$ cells (day 0 , $86.6 \% \pm 5.7 \%$; day $14,74.4 \% \pm 15.6 \%, p=0.0044$ ) was observed, concomitantly with an increase in the percentage of $\mathrm{CD}^{+} \mathrm{O}^{+} \mathrm{B}$ cells (day $0,13.4 \% \pm 5.7 \%$ vs. day $14,25.6 \% \pm 15.6 \%, p=0.0044$ ) (Fig. $1 \mathrm{~b}$ ) that persists during the chronic phase. The percentages and the numbers of CD4 T cells significantly decreased during the acute phase (day $0,53.6 \% \pm 6.5 \%$; day $14,46 \% \pm 11.2 \%, p=$ 0.0460 ) (Fig. 1b). In chronically SIV-infected RMs (day > 180), the percentage of CD4 T cells dropped to $32.6 \% \pm 8.1 \%$, whereas in terms of CD4 T cell counts, three RMs (PB023, PB028, and \#1222) showed lower numbers, due to a lymphopenia, particularly in colic MLNs $\left(1.95 \times 10^{7}, 5.3 \times 10^{7}\right.$, and $8.7 \times 10^{7}$, respectively), which is tenfold lower compared to that observed from the other SIVinfected RMs (PB013, 1.3 × 10 ${ }^{9}$; PB044, 6.6 $\times 10^{8}$; \#2012, 5.2 $\times 10^{8}$; $\# 2070 \mathrm{R}, 6.9 \times 10^{8}$ ). Interestingly, despite lower percentages of CD4 T cells, RMs have even higher numbers of CD4 T cells, due to an increased number of cells recovered from MLNs, compared to healthy RMs (colic MLNs at day $0,3.2 \times 10^{8} \pm 1.43$ vs. at day $>180$, $\left.8.03 \times 10^{8} \pm 3.65, p=0.0095\right)$. Furthermore, our data revealed
(PB013, PB044, \#2012, and \#2070R) higher B-cell counts in RMs in both colic (day $0,6.4 \times 10^{7} \pm 2.8$ vs. $4.510^{8} \pm 3.6, p=0.0048$ ) and ileocolic MLNs (day $0,1.9 \times 10^{8} \pm 1.1$ vs. $5.7 \times 10^{8} \pm 3.8, p=$ 0.019 ) (Fig. 1b). Finally, the percentages of CD8 T cells increased at the chronic phase, compared to healthy RMs (day $0,33.03 \% \pm$ $6.6 \%$; day $>180,41.9 \% \pm 5.4 \%, p=0.0004$ ). Our data revealed that RMs displaying higher CD4 T cell counts also have higher CD8 Tcell numbers in colic MLNs (day $0,2.05 \times 10^{8} \pm 1.1$ vs. $10 \times 10^{8} \pm$ $5.9, p=0.0048$ ) and a trend in ileocolic MLNs (day $0,4.9 \times 10^{8} \pm 4.3$ vs. $10 \times 10^{8} \pm 2.8, p=0.057$ ) (Fig. $1 \mathrm{~b}$ ).

Whereas the viral load in PB023 and PB028 reached $10^{8}$ copies/ $\mathrm{ml}$, only $1.5 \times 10^{6}$ copies $/ \mathrm{ml}$ are detected in RM \#1222, which is similar or lower compared to the other RMs such as PB013 or PB044 $\left(3.5 \times 10^{6}\right.$ copies $/ \mathrm{ml}$ and $5.7 \times 10^{7}$ copies $/ \mathrm{ml}$, respectively); RM \#2012 showing the lowest, $6.3 \times 10^{3}$ copies/ml (Table 1) suggesting that $\mathrm{VL}$ in the blood is not strictly correlated with the extent of CD4 T-cell depletion in MLNs. We have previously reported that MLNs represent a major site for viral replication and stored throughout the course of infection in SIV-infected RMs. ${ }^{16}$ Viral replication in tissues was assessed by in situ hybridization. Diffuse labeling over the follicular dendritic cell (FDC) network in the GC corresponds to virus trapped at the FDC surface (silver grains), whereas individual spots correspond to replicative cells. ${ }^{16,72}$ During the acute phase (day 14) most of the cells are replicative cells (individual spot) (Fig. 2a). In chronically SIVinfected RMs, few SIV RNA cells are detected in RMs with a high level of CD4 T cells in MLNs (SIV RNA cells, $6.4 \pm 3.8$ cells $/ \mathrm{mm}^{2}$ ) and staining corresponds to diffuse labeling in GC (Fig. 2b-d). On the contrary, in RMs having a low level of CD4 T cells, extensive viral replication is detected (SIV RNA cells, $30.1 \pm 16.8$ cells $/ \mathrm{mm}^{2}, p<$ 0.001 compared to the former) (Fig. 2c, d).

Altogether, these results demonstrate the distinct $\mathrm{T}$ - and B-cell dynamics occurring in MLNs of SIV-infected RMs in which the extent of SIV RNA cells is associated with lower levels of CD4 $T$ cells in MLNs.

Early depletion of memory CD4 T cells in MLNs of SIV-infected RMs We then analyzed the dynamics of the CD4 T cell subsets, including Tfh cells. Cells are defined based on the expression of cell surface markers as effector memory (EM: $\mathrm{CD}^{2} \mathrm{~L}^{-} \mathrm{CD} 4 \mathrm{RA}^{-}$), central memory $\left(\mathrm{CM}: \mathrm{CD} 2 \mathrm{~L}^{+} \mathrm{CD} 45 \mathrm{RA}^{-}\right)$, terminally differentiated

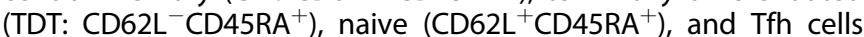
$\left(\mathrm{CXR} 5^{+} \mathrm{CPD} 1^{\text {high }}\right)$ (Fig. 3a). Our results demonstrated an early significant increase in the percentages of naive CD4 T cells (day 0 , $26.2 \% \pm 5.4 \%$; day $14,39.1 \% \pm 12 \% ; p=0.0067$ ) (Fig. $3 b$, left panel). However, their number remained unchanged (Fig. 3b, right panel). On the contrary, we observed a decrease in both the percentage and the number of EM CD4 T cells (day 0, 28.5\% \pm 4.2\%; day $14,18.4 \% \pm 6.3 \% p=0.0006$ ) (Fig. $3 \mathrm{~b}$, left and right panels). Th cells declined in MLNs of SIV-infected RMs at day 30 postinfection (day $0,2.2 \% \pm 0.5 \%$; day $30,1.2 \% \pm 0.8 \%, p=0.0013$ ) (Fig. 3b, left panel). The percentages of CM and TDT were relatively similar in RMs sacrificed at different time points, postinfection. Our data also indicated that the decrease in the percentage of EM compared to healthy RMs in MLNs is similar to the decrease in the percentage observed in the spleen and in Axillary and Inguinal LNs at days 14 and 30 (Fig. 3c), whereas the decrease in the percentage of Tfh cells is higher in the spleen than in MLNs and peripheral LNs (Fig. 3d) suggesting a role of tissue compartmentalization on the early dynamics of EM vs. Tfh cells in SIV-infected RMs

In chronically SIV-infected RMs (day > 180), our results indicated that associated with an increase in the percentage of naïve CD4 $T$ cells, the percentages of both EM and Tfh declined (Fig. 3b, left and right panels). Thus, in SIV-infected RMs (PB023, PB028, and $\# 1222$; low level of CD4 T cells), the percentages of EM in colic MLNs (day $0,27.1 \pm 4.2$ vs. day $>180,12.6 \pm 5.9, p=0.01$ ) and in ileocolic MLNs (day 0, $29.9 \pm 4$ vs. day $>180,13.4 \pm 6.1, p=0.01$ ) 
a

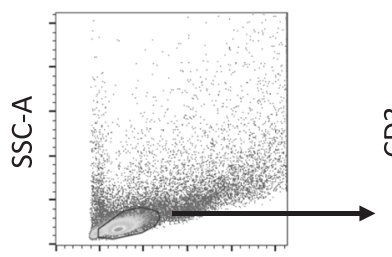

FSC-A

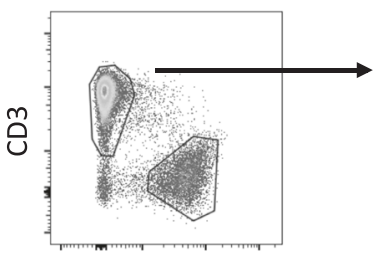

CD20

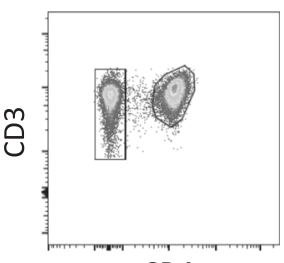

CD4

b
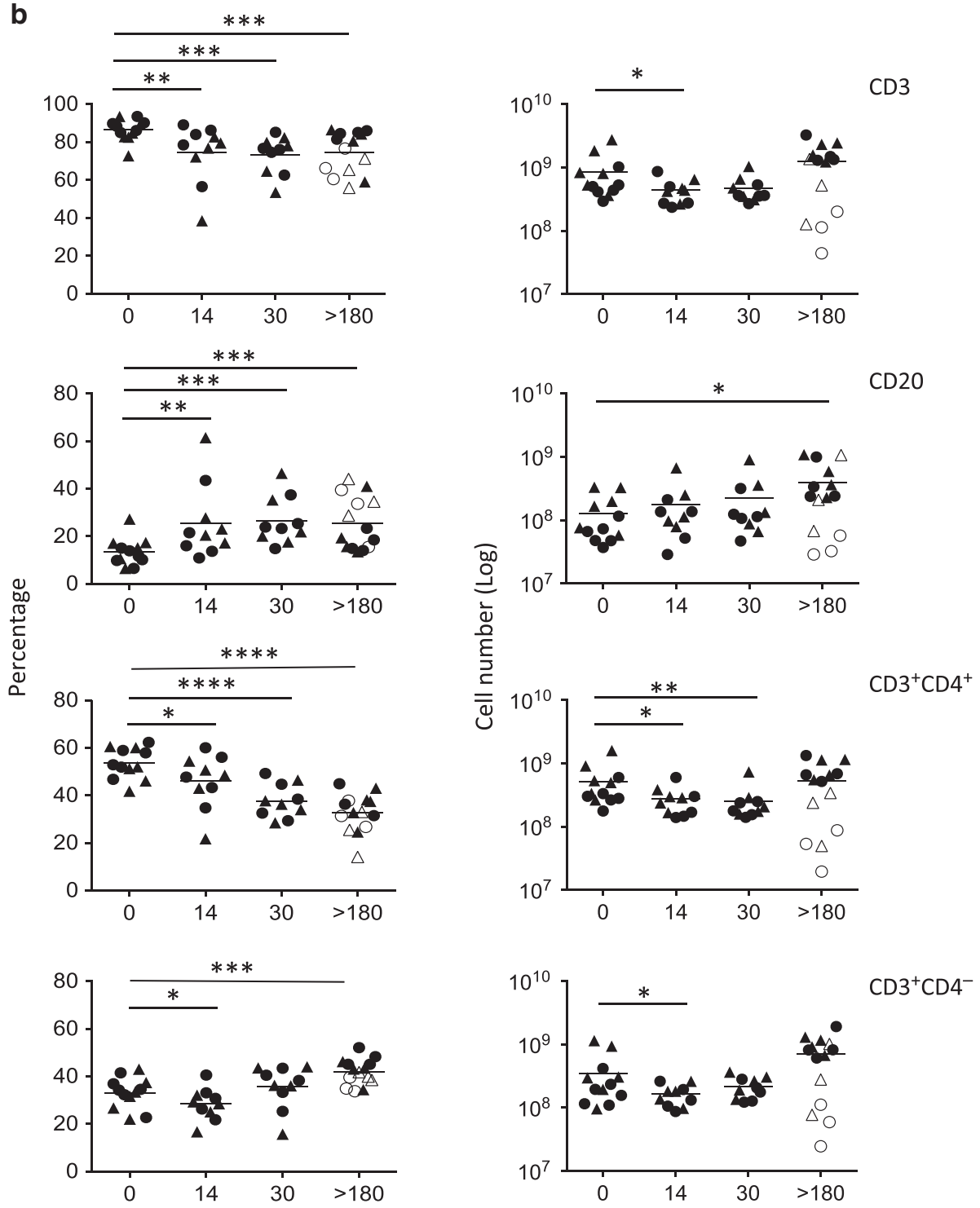

$\mathrm{CD}^{+} \mathrm{CD}^{-}$

Days post-infection

Fig. 1 T- and B-cell dynamics in the MLNs of SIV-infected RMs. a Gating strategy to identify T and B-cell populations in MLNs by flow

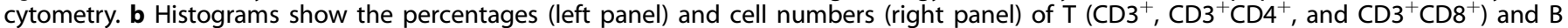
$\left(\mathrm{CD}^{-} \mathrm{CD}^{-} \mathrm{O}^{+}\right)$cells at days $0,14,30$, and $>180$ postinfection. Ileocolic MLNs (triangle) and colic MLNs (circle) are indicated in distinct dots for each RM. At day >180, RMs with low levels of CD4 T cells (PB023, PB028, and \#1222, empty symbols) and RMs with high levels of CD4 (PB013, PB044, \#2012, and \#2070R, full symbols) are shown. Statistical analyses were performed using the Mann-Whitney test. ${ }^{*} p<0.05 ;{ }^{* *} p<0.01$; $* * * p<0.001 ;{ }^{* * * *} p<0.0001$

diminished significantly compared to healthy RMs but despite a trends the difference was not statistically significantly different to the percentage observed in SIV-infected RMs with a high level of CD4 (PB013, PB044, \#2012, and \#2070R) (19.4 44.3 and 21.8 \pm 8.6 , respectively). Similarly, the percentages of Tfh in RMs with low level of CD4 (PB023, PB028, and \#1222) were lower both in colic (day $0,2.04 \pm 0.43$ vs. day $>180,0.75 \pm 0.5, p=0.01$ ) and ileocolic
MLNs (day 0, $2.3 \pm 0.46$ vs. day $>180,0.7 \pm 0.14, p=0.01$ ) compared to healthy RMs and significantly different to the percentages observed in RMs with high level of CD4 (PB013, PB044, \#2012, and \#2070R) (colic, $2.3 \pm 1.4, p=0.05$ and ileocolic, $1.89 \pm 1.18, p=0.02$ ). Finally, in chronically SIV-infected RMs, we observed a decrease in the percentages of EM in the different tissues analyzed (between 20 and $40 \%$ of decrease in the 


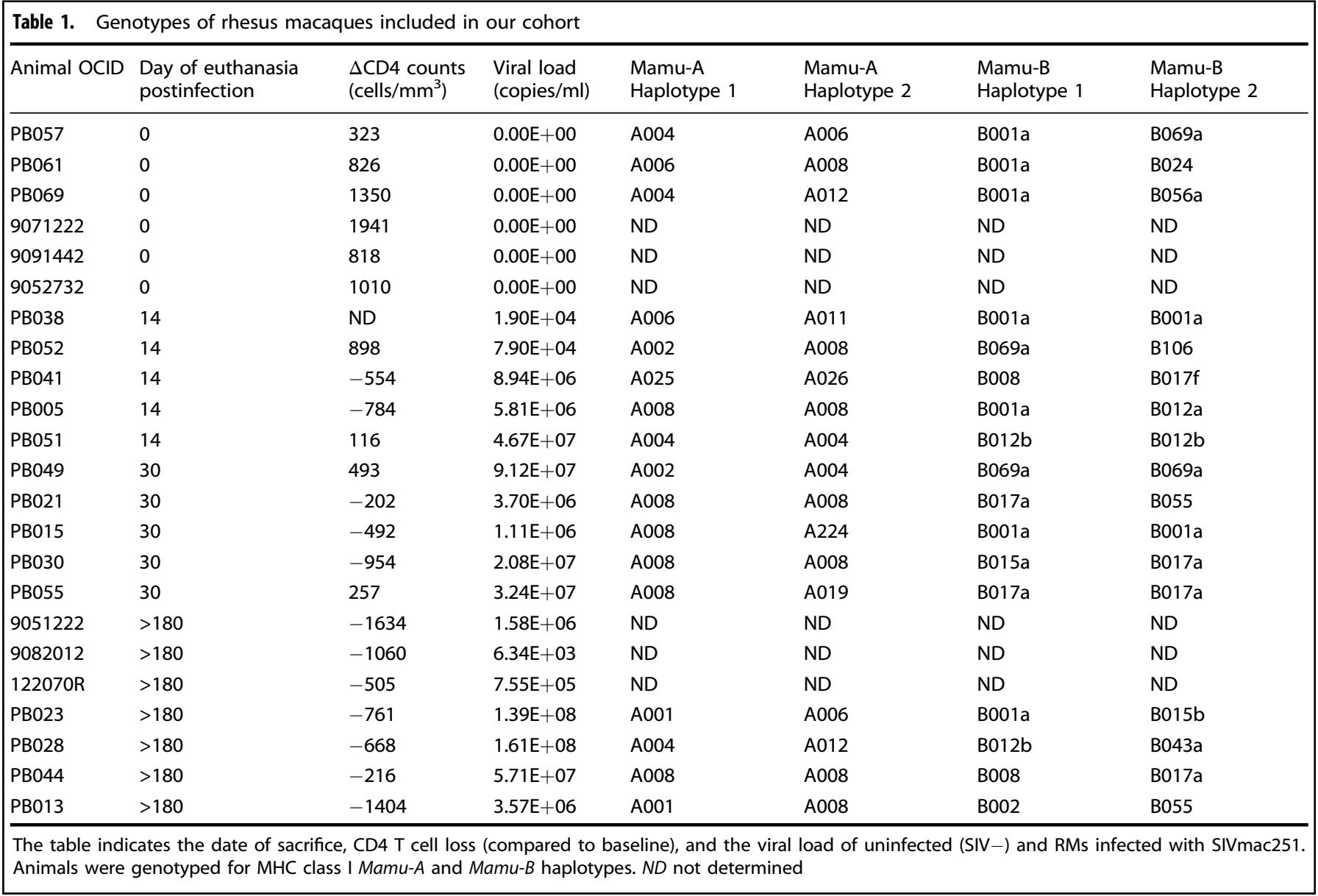

percentage of EM compared to the percentage of EM in healthy RMs) (Fig. 3c), whereas we observed the absence of Tfh decrease in peripheral LNs (axillary and inguinal LNs) or even higher compared to uninfected RMs, but a decreased in MLNs and the spleen (between 20 and $40 \%$ of decrease in the percentage of cells) (Fig. 3d).

Due to lymphopenia (Fig. 1), our results indicated that the number of EM and Tfh cells (Fig. 3b) in colic MLNs were tenfold lower in RMs (PB023, PB028, and \#1222) compared to uninfected RMs (EM: day 0, $9.110^{7} \pm 5.1$ vs. day $>180,7.510^{6} \pm 7.5, p=0.01$ and Tfh: day $0,6.8 \times 10^{6} \pm 3.5$ vs. day $>180,7.6 \times 10^{5} \pm 9.9, p=$ 0.01 ) as well in ileocolic MLNs (EM: day $0,2.1 \times 10^{8} \pm 1.7$ vs. day > $180,3 \times 10^{7} \pm 2.3, p=0.001$ and Tfh: day $0,1.6 \times 10^{7} \pm 1.3$ vs. day $\left.>180,1.6 \times 10^{6} \pm 7.1, p=0.01\right)$. In contrary, in RMs with high levels of CD4 (PB013, PB044, \#2012, and \#2070R), nor the number of EM or the number of Tfh are significantly decreased in colic (EM: $1.6 \times$ $10^{8} \pm 1$ and Tfh: $\left.1.7 \times 10^{7} \pm 1.9, p=0.001\right)$ and in ileocolic MLNs (EM: $1.7 \times 10^{8} \pm 3.2$ and Tfh: $1.3 \times 10^{7} \pm 0.8$ ) compared to uninfected RMs.

Altogether, our results indicate a decrease in EM and Tfh cell subsets during the acute phase of the infection, which is more drastic in chronically SIV-infected RMs with low levels of CD4 $T$ cells in MLNs.

IL-21 and CXCL13 expressions in the MLNs of SIV-infected RMs Having observed that SIV is present in GC, whereas Tfh cells are diminished, we then assessed by confocal microscopy Tfh cell distribution and its relationship with GC remodeling in the MLNs of SIV-infected RMs. Two weeks after an SIV infection, an enlargement of B-cell follicles can be observed in MLNs (Fig. 4a,
C and Supplementary Fig. 1) compared to healthy RMs (Fig. 4a-c). This enlargement of the size increases in chronically SIV-infected RMs (Fig. 4a-c). However, due to the MLN adenopathy, their numbers per $\mathrm{mm}^{2}$ of tissue are decreasing. Tfh cells $\left(\mathrm{CD} 4^{+} \mathrm{CXCR} 5^{+} \mathrm{PD}-1^{+}\right)$are detected in the B-cell follicles of MLNs derived from healthy RMs (Fig. 4Aa), whereas they are hardly detectable in the B-cell follicles in MLNs of RMs with low levels of CD4 T cells (Fig. 4Ac) compared to RMs with high levels of CD4 cells (Fig. 4Ad). IL-21 characterizes Tfh cells. From the same tissue sections, we then assessed the levels of IL-21 in MLNs and observed a lower level of IL-21 in SIV-infected RMs, compared to healthy RMs (Figs $4 b-d$ ). The level of IL-21-producing cells was lower in SIV-infected RMs with low CD4 T cells, which is consistent with the depletion of Tfh cells assessed by flow cytometry. T-cell distribution in lymphoid tissues depends in part on the presence of the expressed chemokine. It is well-known that FDC produces CXCL13, the ligand of CXCR5. We consistently found in healthy RMs that CXCL13 is expressed mainly in the GCs of the MLNs (Fig. 4Ba). Our results highlighted changes in the distribution of CXCL13-expressing cells along the B-cell follicle (Fig. 4b) that we quantified by using the kurtosis index (Fig. 4e). Thus, instead of mostly labeling the GCs in healthy RMs, staining is scattered in SIVinfected RMs. Interestingly, this change occurs early after infection (Fig. 4b-e). Moreover, in SIV-infected RMs with low levels of CD4 $T$ cells (Fig. $4 \mathrm{BC}$ ), stained cells seem to be more related to perivascular cells. $^{73}$

Altogether, these results demonstrate a remodeling of the MLNs architecture associated with a change in the expression of CXCL13 in SIV-infected RMs with a low level of CD4 with a decline in IL-21 expressing cells. 
a

b
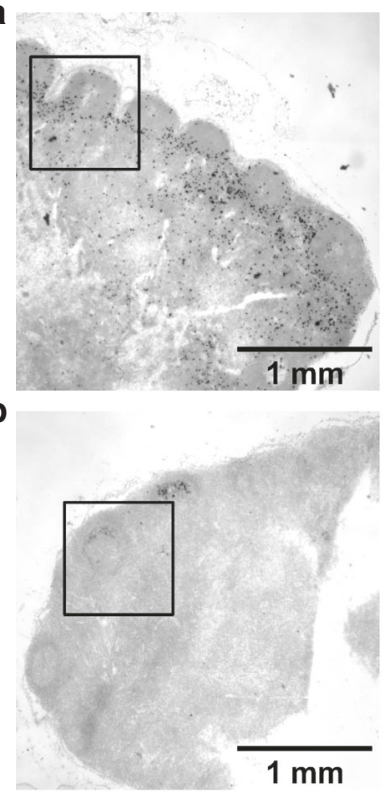

C

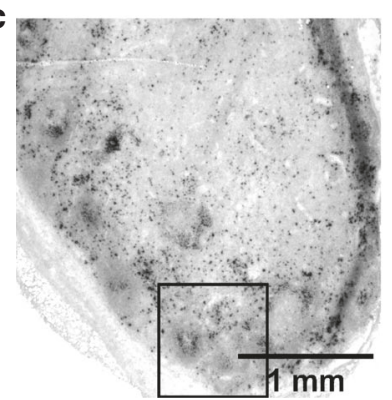

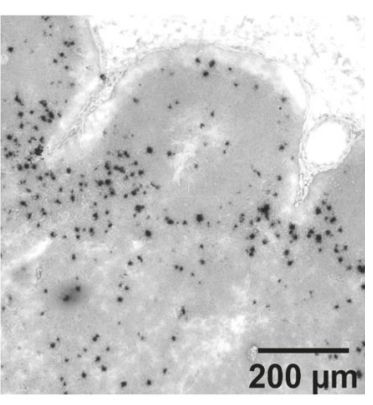
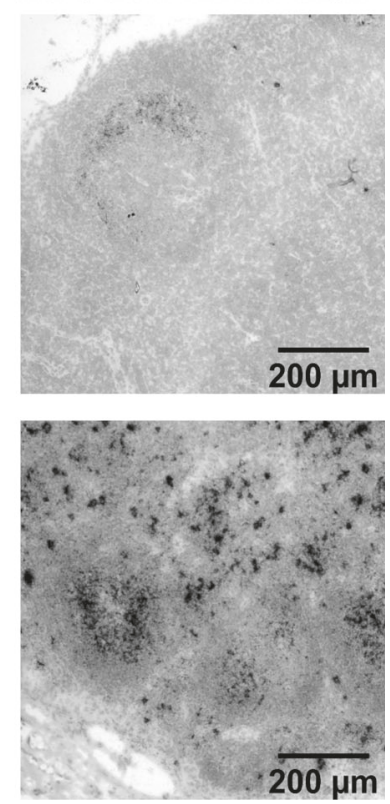

d

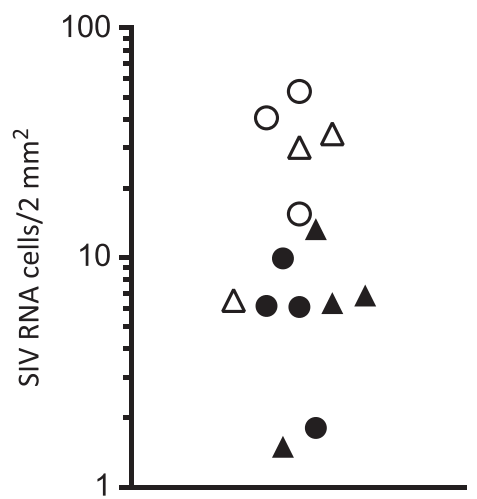

Fig. 2 Detection of viral RNA cells in the MLNs of SIV-infected RMs. SIV RNA ${ }^{+}$cells in LNs are detected by in situ hybridization a at day 14 and b, c in chronically SIV-infected RMs with either high (b) or low (c) levels of CD4 T cells (Scale bars are included). d lleocolic MLNs (triangle) and colic MLNs (circle) are indicated in distinct dots for each chronically SIV-infected RM (open and closed dots are low and high levels of CD4 T cells, respectively)

Depletion of memory B cells expressing CD95 in the MLNs of SIVinfected RMs

Because we observed lower Tfh cell numbers in the MLNs of SIVinfected RMs, which are considered to be essential for B-cell differentiation with a CXCL13 staining, which is generally observed during late embryonic splenic vasculature in which $B$ cells are essentially immature, ${ }^{73}$ we analyzed the B-cell differentiation. Bcell subsets are defined as follow: naive $\left(\mathrm{CD} 21^{+} \mathrm{CD} 27^{-}\right)$, resting memory (RM: $\quad \mathrm{CD} 21^{+} \mathrm{CD} 27^{+}$), activated memory (AM:

$\mathrm{CD} 21^{-} \mathrm{CD} 27^{+}$), and tissue memory B cells (TM: CD21 ${ }^{-} \mathrm{CD} 27^{-}$) (Fig. 5a). It was previously reported that in healthy RMs, the relative frequencies of B-cell subsets differed substantially from blood to peripheral LNs, and upon infection, a significant decrease in the frequency of naive $B$ cells from peripheral $L N s$ is compensated by an increase in $\mathrm{CD} 27^{+}$memory B cells. ${ }^{74}$ In MLNs, no major changes were observed in the distribution of B-cell subsets during the acute phase of infection (Fig. 5b). At day 30 postinfection, we observed that the percentages of AM $\left(\mathrm{CD} 21^{-} \mathrm{CD} 27^{+}\right)$and $\mathrm{RM}\left(\mathrm{CD} 21^{+} \mathrm{CD} 27^{+}\right) \mathrm{B}$ cells in MLNs are slightly decreased compared to the percentage of $B$ cells from healthy RMs (AM: day 0, 13.4\% $\pm 4.2 \%$; day 30,8.9\% $\pm 4.6 \% p=0.039$, and RM: day 0, 28.4\% $\pm 5.7 \%$; day 30, 23.7\% $\pm 5.8 \%, p=0.01$ ) (Fig. $5 b$ ). In chronically SIV-infected RMs ( $>180$ days), these percentages of AM and RM B cells were lowered reaching 5.9\% $\pm 3.4 \%(p<0.001)$ and $20.5 \% \pm 8.8 \%(p=0.004)$, respectively (Fig. $5 b)$. Conversely, the percentage of naive $B$ cells increased (day $0,42.4 \% \pm 8.2 \%$; day $180,55.2 \% \pm 9.2 \%, p<0.001)$. Because, as shown in Fig. $1 \mathrm{~b}$, our data indicated that the percentages of $B$ cells increase at day 180 in MLNs (Fig. 1b), we reevaluated these percentages accordingly. Thus, the percentage of naive $B$ cells increased, particularly in RMs (PB023, PB028, and \#1222) with low levels of CD4 (19.7\% \pm 9.1$)$, whereas this percentage was only $10.4 \% \pm 4.2 \%(p<0.02)$ in RMs with high levels of CD4 (PB013, PB044, \#2012, and \#2070R). Thus, the fraction of naive $B$ cells in MLNs increased in the former. The percentages in the other B-cell subsets were not significantly different between both groups of RMs. We then assessed the correlation between Tfh and B-cell subsets in colic and ileocolic MLNs (Fig. 6). We observed a negative correlation between the percentages of naive $B$ cells and Tfh cells, and a positive correlation with AM B cells (Fig. 6). We also evaluated Fas (CD95) expression, which is highly expressed on GC B cells, ${ }^{75,76}$ and contributes to the regulation of $B$ cells. ${ }^{7,78}$ We observed a significant decrease in the percentage of $B$ cells expressing Fas during the acute (day $0,16.1 \% \pm 2.9 \%$; day $14,12.6 \% \pm 5.6 \% p=$ 0.0126 ) and the chronic phases of SIV infection (day $>180,7.1 \% \pm$ $2.2 \% p=0.0001$ ) (Fig. 5c), indicating the decrease of GC B cells in SIV-infected RMs. Fas is mainly expressed on RM $(49.3 \% \pm 9.4 \%)$ and AM B cells $(72.5 \% \pm 10.2 \%)$ in healthy RMs, whereas only $15.6 \% \pm 4.8 \%$ of TM expressed Fas, and less than $5 \%$ of naive cells. At the chronic phase, these percentages decreased to $40.9 \% \pm$ $14.2 \%(p=0.03)$ and $58.5 \% \pm 17.9 \%(p=0.01)$ for RM and AM B cells, respectively. By analyzing the pool of B cells expressing CD95 (Fig. $5 \mathrm{~d}$ ), we found that the numbers of RM and AM B cells expressing Fas are reduced in PB023, PB028, and \#1222 (RM B cells, $301 \pm 105$ vs. $998 \pm 667$ in healthy RMs, $p=0.0002$; AM B cells, $66.7 \pm 39.3$ vs. $610 \pm 353$ in healthy RMs, $p<0.0001$ ), whereas these numbers were higher in RM B cells of PB013, PB044, \#2012, and \#2070R (3841 \pm 364 vs. $998 \pm 667$ healthy RMs, $p=0.002)$ and not significantly different for AM B cells $(2299 \pm 2380$ vs. $610 \pm 353$, $p=\mathrm{ns})$.

Altogether, our results strongly suggested that AM and GC B cells are mostly affected by the absence of Tfh cells, and their depletion characterized animals having low CD4 T cells in MLNs.

Commutation of effector memory MLN Tfh cells toward central memory is associated with T-bet expression and Stat5 phosphorylation in SIV-infected RMs

Several TFs, including activator factors such as Bcl6, c-Maf, and TCF-1, $42-46,49-52$ and repressor TFs, such as KLF2, Foxo1, and Tbet ${ }^{53,54}$ have been reported to play a major role in regulating Tfh cell differentiation. Our results demonstrated a transient increase in the expression of KLF2 and Foxo1 at days 14 and 30, along with an increase in the expression level of T-bet in both colic and ileocolic MLNs (Fig. 7a, b). Bcl6 is slightly increased at day 30, whereas cMaf expression increased at day $>180$ only in colic MLNs. In addition to CMaf, TCF1, which is essential for both the initiation of differentiation and the effector function of Tfh cells, is 


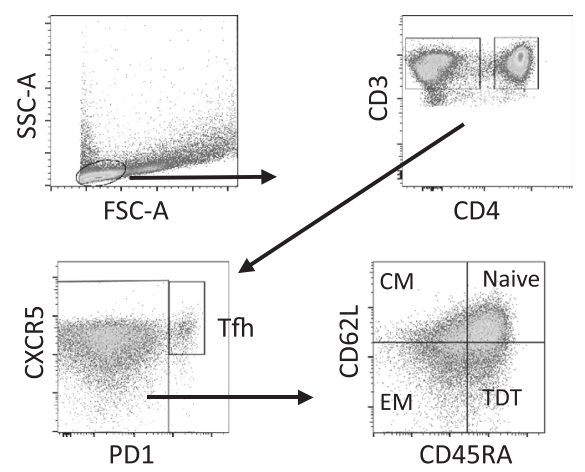

b
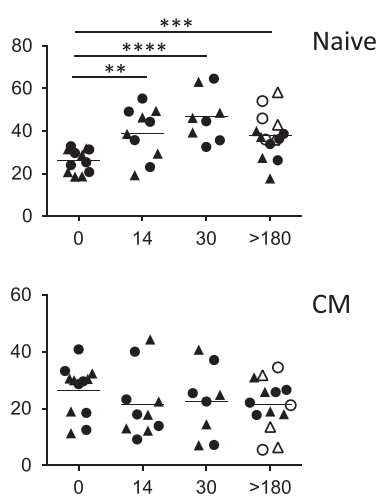

$\mathrm{CM}$
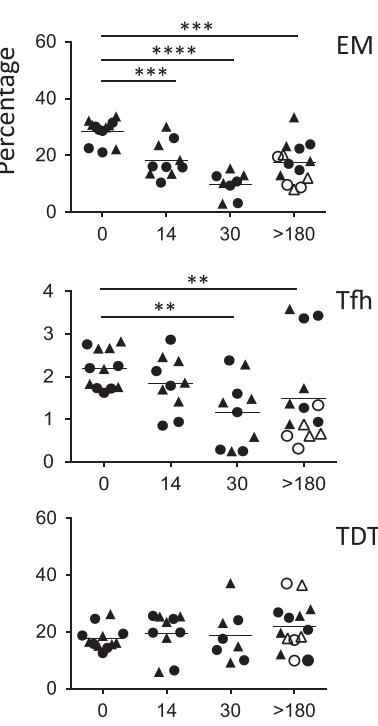

TDT

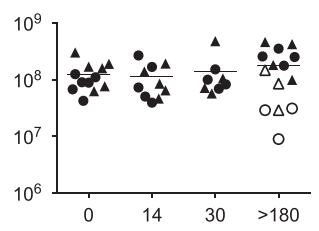

Days post-infection

C
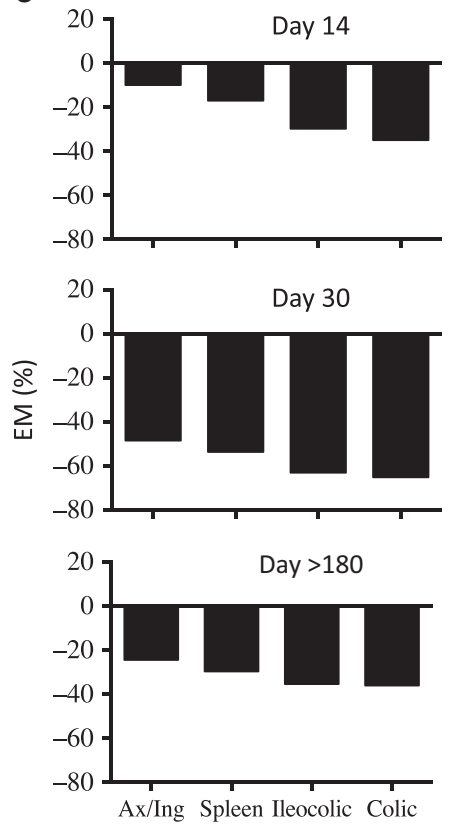

d
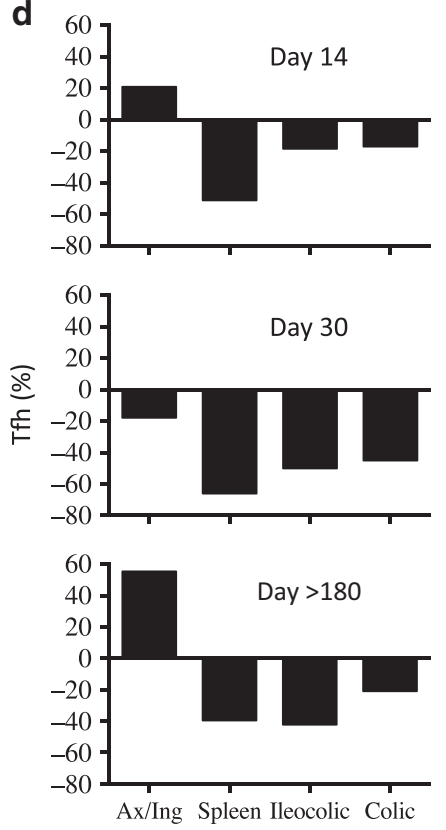

Fig. 3 Early depletion of Tfh and of memory CD4 T cells in the MLNs of SIV-infected RMs. a Gating strategy to identify CD4 T cell subsets

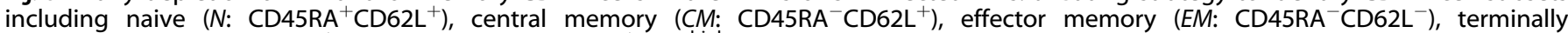
differentiated (TDT: $\left.C D 45 \mathrm{RA}^{+} \mathrm{CD} \mathrm{L}^{-}\right)$and Tfh $\left(\mathrm{CXCR5}{ }^{+} \mathrm{PD}-1^{\text {high }}\right)$. b Percentages (left panel) and cell numbers (right panel) of CD4 T-cell subsets are shown as described in Fig. 1 at days $0,14,30$, and $>180$ postinfection. $\mathbf{c}$, $\mathbf{d}$ Histograms show the percentage of decrease in the percentages of EM and Tfh CD4 T-cell subsets compared to healthy RMs at days 14, 30, and >180 postinfection (mean \% of EM at day 0 minus the mean $\%$ of EM at days postinfection/by the mean $\%$ of EM at day 0$) \times 100$. Both axillary/inguinal (Ax/Ing) LNs, ileocolic and colic MLNs, and spleen are shown. Statistical analyses were performed using the Mann-Whitney test. ${ }^{*} p<0.05 ;{ }^{* *} p<0.01 ;{ }^{* * *} p<0.001 ;{ }^{* * * *} p<0.0001$

increased only at day 14 post-infection (Fig. 8a, b). Because KLF2 and Foxo1 regulate the expression of $C D 62 L, 55,56$ we measured the ratio of effector memory $\left(\mathrm{CD} 2 \mathrm{~L}^{-} \mathrm{CD} 45 \mathrm{RA}{ }^{-}\right)$vs. central memory $\left(\mathrm{CD} 2 \mathrm{~L}^{+} \mathrm{CD} 45 \mathrm{RA}^{-}\right)$phenotype. Our results indicated that Th cells switch from an effector memory phenotype in healthy RMs ( $r=1.8$ at day 0$)$ to a central memory phenotype after SIV infection (at day 14, $r=0.93$; at day 30, $r=0.88$ ) (Fig. 8c). Thus, our results suggest that early after infection, inhibitory TFs are increased that may impact on Tfh differentiation.
We then analyzed the phosphorylation status of Stat5, which has been associated with a block in the differentiation of Tfh cells. $^{43,44}$ Interestingly, early after SIV infection and thereafter, Stat5 phosphorylation increases and remains elevated in Tfh cells (Fig. 8a, b). Because IL-7 induces Stat5 phosphorylation and has been reported to repress Tfh cell differentiation, ${ }^{59}$ we investigated the cell surface expression of IL-7 receptor (CD127) on Tfh cells (Fig. $8 \mathrm{~d}$, e). Our results revealed a significant and progressive increase in the percentage of Tfh cells expressing CD127 in MLNs 
a
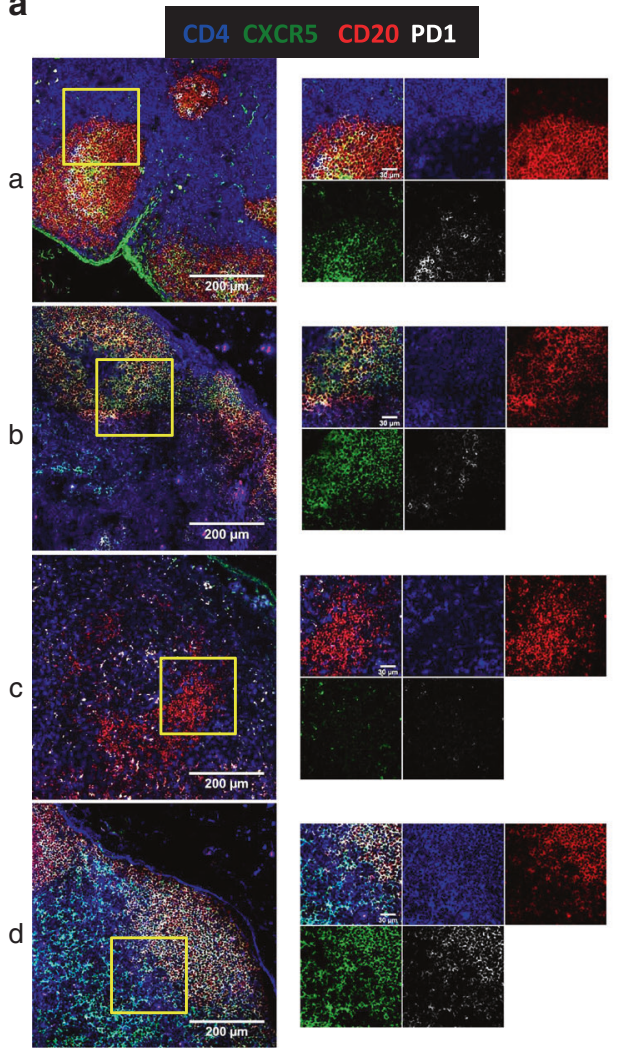

b
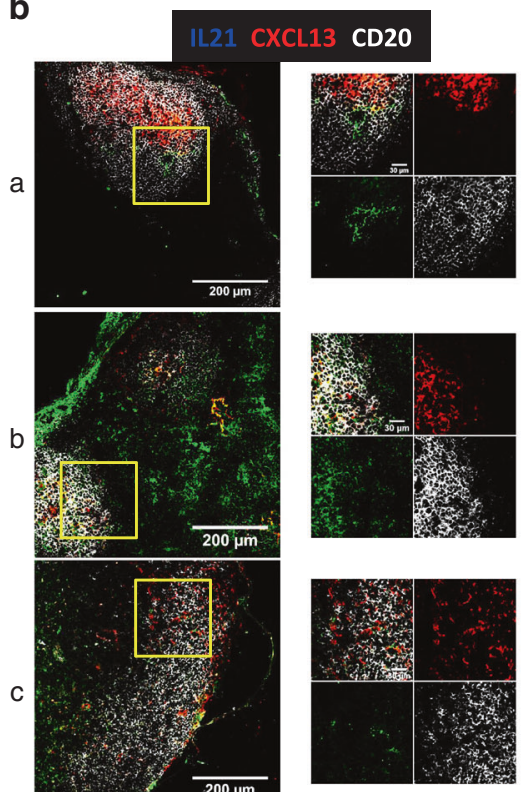

c
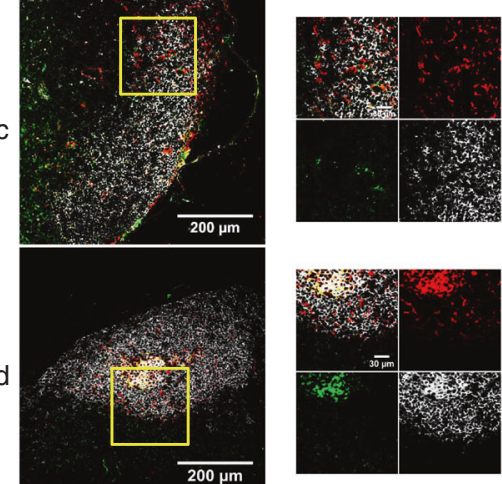

C

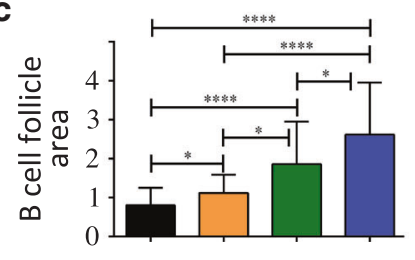

Naive d

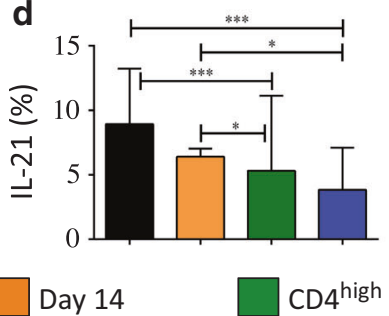

e

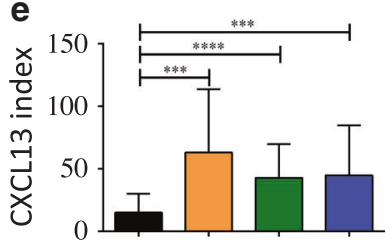

CD4 $4^{\text {low }}$

Fig. 4 Distribution of Tfh cells in the MLNs of SIV-infected RMs. a MLN sections were stained with antibodies against CD4 (blue), CD20 (red), CXCR5 (green) and PD1 (white) and b against IL21 (green), CXCL13 (red) and CD20 (white). Tissue sections are imaged by confocal microscopy. Representative pictures of naive a) and SIV-infected RM at days 14 (b) and $>180$ (c, d) in chronically SIV-infected RMs (CD4 ${ }^{\text {low }}$ (c) vs. CD4 ${ }^{\text {high }}$ (d)). Higher magnification is shown on the right part of the picture. Scale bars are included. c B-cell follicle area quantification. Area of B-cell follicles was performed by using Image J software. About 17-26 B-cell follicles were measured for each tissue section, and 3-5 tissue sections were analyzed for each RM. Three RMs were analyzed at each time point. A representative picture of follicle quantification is shown in Supplementary Fig. 1. d IL-21 quantification. IL-21 was quantified on the same tissue sections, and the results shown represent the percentage of IL-21 staining per follicle area. Representative picture of IL-21 quantification is shown in Supplementary Fig. 2. e CXCL13 distribution within B-cell follicle. CXCL13 distribution in the B-cell follicles was evaluated by the Kurtosis index using Image J software, which is a descriptor of the shape of the biodistribution. A Kurtosis index of 3 is considered as a normal distribution. Representative picture of CXCL13 quantification is shown in Supplementary Fig. 3. Statistical analyses were performed using the Mann-Whitney test. ${ }^{*} p<0.05 ;{ }^{* *} p<0.01 ;{ }^{* * *} p<0.001 ;{ }^{* * * *} p<$ 0.0001

during the acute (day $0,6.3 \% \pm 1.2 \%$; day $30,21.3 \% \pm 5.6 \% ; p<$ 0.0001 ) and the chronic phases of SIV infection (day $>180,18.3 \%$ $\pm 7.8 \% ; p<0.0001$ ) (Fig. 8e).

Altogether, our results demonstrate that Tfh cells from MLNs are associated with a dysregulation of the TF network early after SIV infection, switching from EM to $\mathrm{CM}$ associated with Stat5 phosphorylation.

SIV infection leads to the downregulation of mRNAs coding for the members of the IL-12 cytokine family

Several groups have reported that IL-12, a cytokine driving the Th1 response, induces IL-21 expression in human CD4 T cells, ${ }^{60,61}$ whereas the impact of IL-27 on Tfh cells seems to be speciesdependent. $^{61,65-67}$ Formation of $\mathrm{IL}-12$ is related to mRNA expression coding for the p40 $\beta$ chain and the p35 a chain, whereas IL-27 is formed by the association of the Ebi3 $\beta$ chain with the p28 a chain. The $p 40 \beta$ chain can also pair with the p19 a chain forming the IL-23, while the Ebi3 $\beta$ chain with the p35 a chains can form IL-35 family of interleukins (Fig. 9a). In the context of HIV-infected individuals, other groups and ours have previously reported a defect in the p40 and p35 mRNAs forming the IL-12., ${ }^{2,71,80}$ We thus decided to quantify the relative levels of mRNA expression coding for these different $\alpha$ and $\beta$ chains that form the IL-12 cytokine family. Indeed, the protein-detecting tools (antibodies and ELISA), particularly IL-27 and IL-35, do not cross-react for monkeys. Consistent with our previous results, we observed lower levels of the mRNA encoding for p40 and p35 in MLNs of RMs with low 


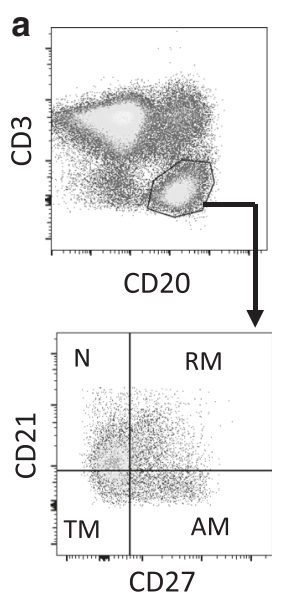

b
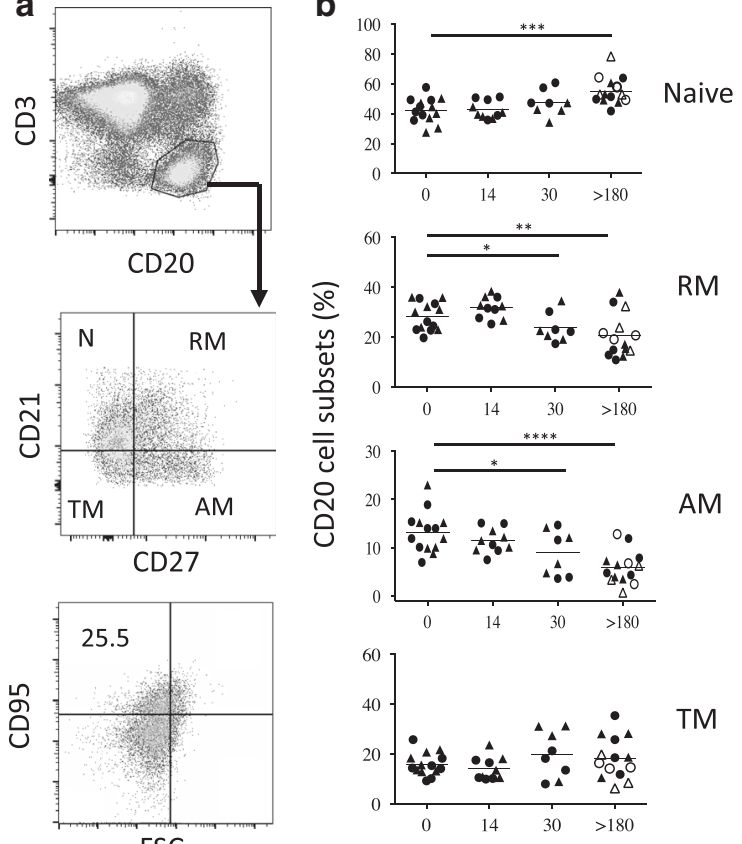

FSC

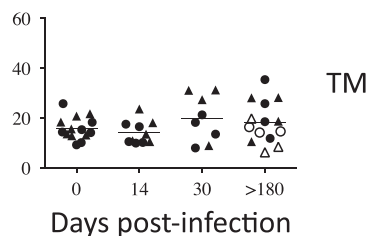

C

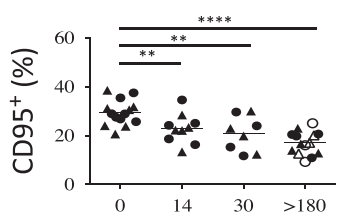

d

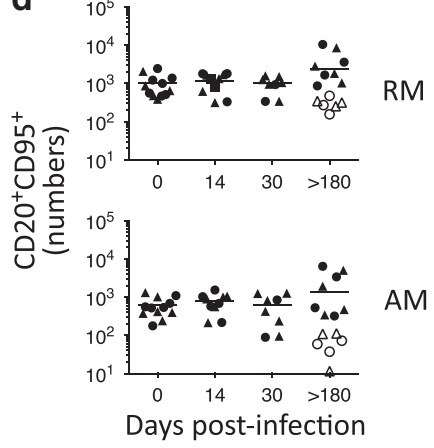

Fig. 5 B-cell dynamics in the MLNs of SIV-infected RMs. a Gating strategy to identify B-cell subsets, including naive (N: $\mathrm{CD}^{2} 1^{+} \mathrm{CD} 27^{-}$), resting memory (RM: $\mathrm{CD} 21^{+} \mathrm{CD} 27^{+}$), activated memory (AM: $\mathrm{CD} 21^{-} \mathrm{CD} 27^{+}$) and tissue memory (TM: $\mathrm{CD} 21^{-} \mathrm{CD} 27^{-}$) B cells. Furthermore, the expression of Fas (CD95) was assessed by flow cytometry. b Percentages of B-cell subsets are shown at days $0,14,30$, and $>180$ postinfection, as described in Fig. 1. c Histograms show the percentage of B cells expressing CD95. d B-cell numbers of RM and AM subsets expressing Fas (CD95) are shown as described in Fig. 1. Statistical analyses were performed using the Mann-Whitney test. ${ }^{*} p<0.05$; ${ }^{* *} p<0.01$; ${ }^{* * *} p<0.001$; $* * * * 0<0.0001$

levels of CD4 cells compared to healthy RMs (p40, $-4.6 \pm 0.64$ vs. $-2.5 \pm 0.56, p=0.02$ and $\mathrm{p} 35,-6.5 \pm 0.65$ vs. $-3.3 \pm 0.26, p=$ 0.001 , respectively) (Fig. 9b). Our results also indicated a lower level of the p19 mRNA between both groups of SIV-infected RMs (p19, $-3.1 \pm 0.72$ vs. $-1.4 \pm 0.10, p=0.05$ ). On the contrary, no significant difference was observed in the levels of mRNA coding for EBi3 and p28 mRNA in SIV-infected RMs compared to healthy RMs (Fig. 9b). These results indicated that SIV infection is associated with lower transcripts that form IL-12, IL-23, and IL-35 in the MLNs of monkeys with low levels of CD4 T cells, compared to healthy or RMs with high levels of CD4 T cells (Fig. 9c).

Thus, our results indicate a major decline in the expression of several transcripts required to form several IL-12 family members in MLNs, but not for those coding for IL-27.

IL-27 induces inhibitory TFs and T-bet expression in Tfh cells from the MLNs of RMs

It has been reported that IL- 6 is essential to induce IL-21 and sustain Tfh cells. ${ }^{81-83}$ The IL-6 receptor is formed by the association with the IL- 6 receptor and the gp130. This latter is shared with the IL-27 receptor ${ }^{84}$ involving the WSX-1/T (homologous to the IL-12Rß2 subunit) suggesting that IL-6 and IL-27 may be competitive for gp130. Therefore, we decided to evaluate the impact of IL-27 on Tfh cells derived from MLNs in comparison to IL-2 and IL-7 reported to antagonize Tfh cell differentiation through Stat5 phosphorylation. ${ }^{60,61}$ Because Tfh cells are subsequently lost in the absence of $B$ cells, ${ }^{85}$ we used MLN cell suspension instead of purified T cells. MLNs are stimulated in the presence of low concentrations of coated CD3 and CD28 mAbs and incubated in the absence or presence of IL- 6 and IL-21 to support the growth of Tfh cells, and in the absence or presence of $\mathrm{IL}-2, \mathrm{IL}-7$, or IL-27. Whereas the mean fluorescence intensity of CXCR5 is increased at day 5 in the presence of either IL6 or IL-6/IL21 , our results indicated that IL-2, IL-7, and IL-27 downregulated
CXCR5 expression (Fig. 10a). IL-6/IL-21 increased the percentage and the number of Tfh cells (CXCR5 ${ }^{+} \mathrm{PD} 1^{\text {high }}$ ) compared to T-cell stimulation alone (Fig. 10b, c). On the contrary, IL-7 or IL-27 decreased both the percentage and the number (Fig. 10b). Our results highlighted that IL-27 increases the percentage (Fig. $8 \mathrm{~b}$ ) and the number (Fig. 10c) of the CXCR5 ${ }^{\text {low }} \mathrm{PD} 1^{\text {high }}$ population.

We then analyzed in more detail the impact of interleukins on the levels of TFs in CXCR5 ${ }^{+}$PD1 ${ }^{\text {high }}$ (Fig. 11a). Because the expression of CXCR5 is downregulated we also extended the analysis of TFs expressions in CXCR5 ${ }^{\text {low } P D} 1^{\text {high }}$ (Fig. 11b). In comparison to cells stimulated with CD3/CD28 only, IL-6 increased the percentage of cells expressing TCF1 and the levels of cMaf in CXCR5 ${ }^{+} \mathrm{PD} 1^{\text {high }} \mathrm{T}$ cells consistent with the increase in Th cells (Fig. 11a). The addition of IL-21 with IL-6 increased TCF1 but also KLF2. The levels of the two TFs, Foxo1, and T-bet, in CXCR5 ${ }^{+} \mathrm{PD} 1^{\text {high }}$ cells are increased in the presence of IL-2 and IL-7 that is associated with Stat5 phosphorylation (pStat5) compared to IL-6/IL-21 (Fig. 11a). Most importantly, our results highlighted that IL-27 upregulates the expression of Foxo1, T-bet, and pStat 5 in $\mathrm{CXCR} 5^{+}$PD $1^{\text {high }}$ cells (Fig. 11a). IL-27 was more efficient to increase the level of T-bet expression than that observed in the presence of IL-2 or IL-7. However, the expression of KLF2 is not modulated in CXCR5 ${ }^{+} \mathrm{PD} 1^{\text {high }}$ cells compared to IL6/IL-21 (Fig. 11a). These results demonstrated that IL-27 is a potent cytokine in inducing a Th1 profile in this Tfh cell subset. Our results also highlighted that IL-2, IL-7, and IL-27 enhanced the levels of Foxo1 and T-bet in CXCR5 ${ }^{\text {low }}$ PD $1^{\text {high }}$ cells (Fig. $11 \mathrm{~b}$ ) as well of KLF2. IL-2 and IL-27 induced the downregulation of TCF1 compared to IL-6/IL-21 in CXCR5 ${ }^{\text {low } P D} 1^{\text {high }}$ cells (Fig. $11 \mathrm{~b}$ ).

Altogether, our results highlighted that IL-27 not only induces the expression of TFs, which are inhibitory of Tfh cell differentiation but is also more potent than IL-2 or IL-7 in inhibiting mucosal Tfh cells. 

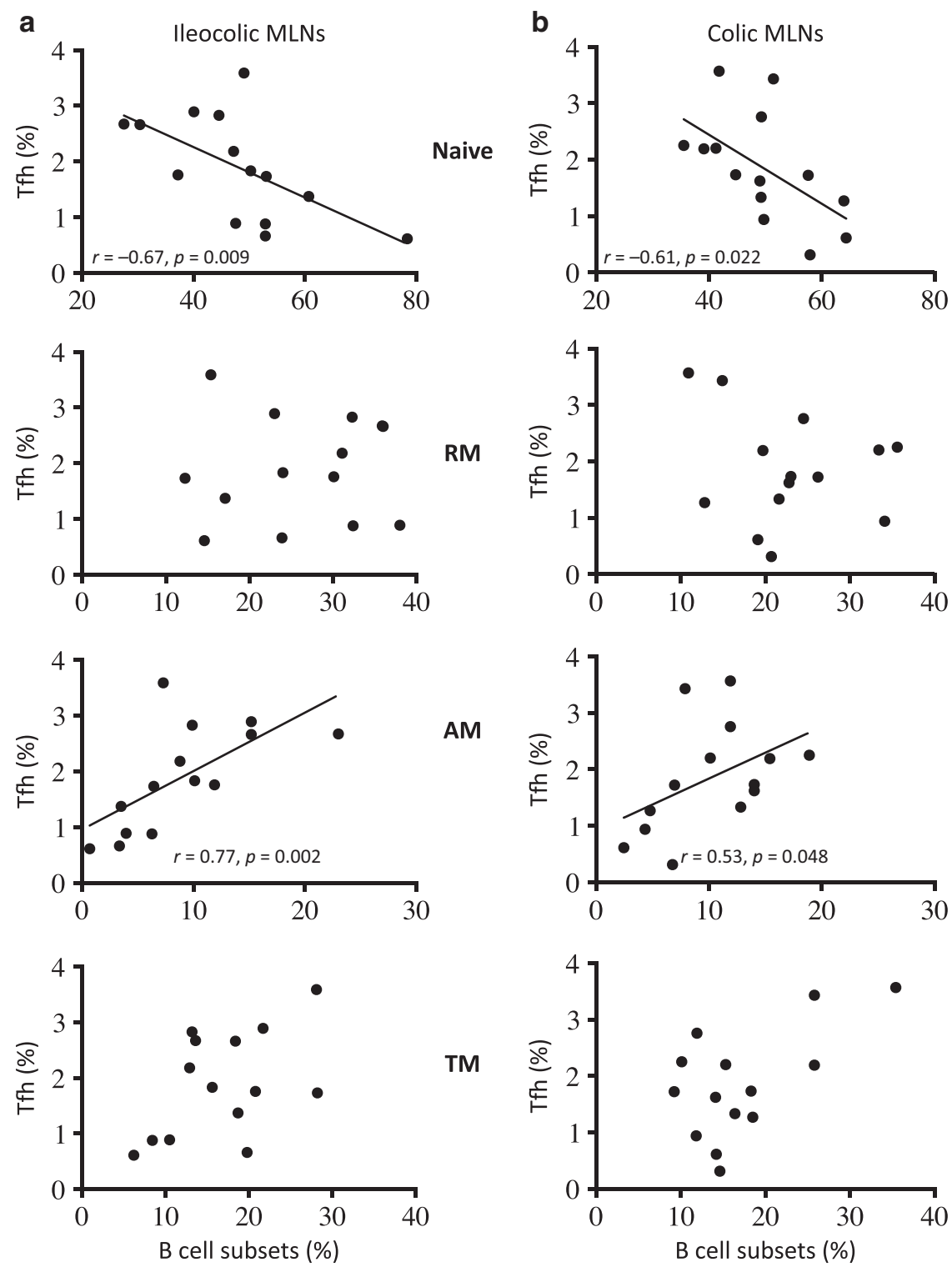

Fig. 6 Correlation between Tfh cells and memory B cells. Tfh were plotted against B-cell subsets in either ileocolic or colic MLNs. Each dot represents an individual RM at the time of death. Spearman analysis was used for correlations. The $r$ and $p$ values are indicated in the figures

\section{DISCUSSION}

In this study, we demonstrated that IL-27, a member of the IL-12 cytokine family, is critical to promote in vitro Th1-like Tfh cells from MLNs, and to induce the expression of inhibitory TFs and Stat5 phosphorylation reported to negatively regulate Tfh cell differentiation. Tfh cells derived from MLNs of SIV-infected RMs display a similar profile early after infection. Our results also highlighted in RMs with low levels of CD4 T cells in MLNs with mRNAs coding for the $\alpha$ and $\beta$ chains forming the IL-12, IL-23, and IL-35 are decreased, whereas those forming the IL-27, EBi3, and p28 are unchanged. Furthermore, our results indicated the loss of mucosal Tfh cells along with the loss of memory B cells and GC B cells expressing CD95 in SIV-infected RMs. Because MLNs are critical components of the GALT and the largest in the body, maintaining oral tolerance, ${ }^{86-88}$ this early abnormal differentiation and depletion of CD4 T cells in these inductive sites (MLNs) could provide a rationale to the absence of replenishment of these effector cells in the lamina propria, the main effector sites, which therefore may contribute to the inability of the immune system to control HIV and SIV infections in mucosal tissues. ${ }^{9,10,14,89-92}$
During LCMV infection in mice, it was been reported that IL-27 induced IL-21 from CD4 T cells, whereas IL- 6 is required to sustain Tfh cells. ${ }^{6,82}$ In particular, FDCs produce IL-6, supporting GC reactions during immunization ${ }^{66,82,93}$ consistent with the observation that the absence of IL- 6 is associated with an early defect in Tfh cells. ${ }^{81}$ Whereas IL-27 induces in vitro IL-21 secretion from murine naive $T$ cells, ${ }^{65,67} \mathrm{IL}-27$ was fourfold less potent than IL-12 to induce IL-21, and IL-27 had little effect on Bcl-6 mRNA expression, suggesting that IL-27 did not induce full Tfh differentiation. ${ }^{65}$ Other studies indicate that only IL-12, and not IL-27, induced IL-21 expression from human cells. ${ }^{61}$ Thus, the impact of IL-27 in the genesis of Th cells was proposed to be species-dependent, ${ }^{61}$ which may have considerable implications for B-cell vaccine development in which Tfh cells are critical. Our results in monkeys demonstrated that in vitro IL-6, in combination with IL-21, increases Tfh cells, whereas IL-27 impacts on this pool by downregulating the expression of CXCR5, similarly as IL-2 and IL-7. Our results also indicated that IL-27 increases T-bet, which is consistent with earlier reports ${ }^{94,95}$ but also induces Stat5 phosphorylation, similarly to IL-2 and IL-7 reported to negatively 

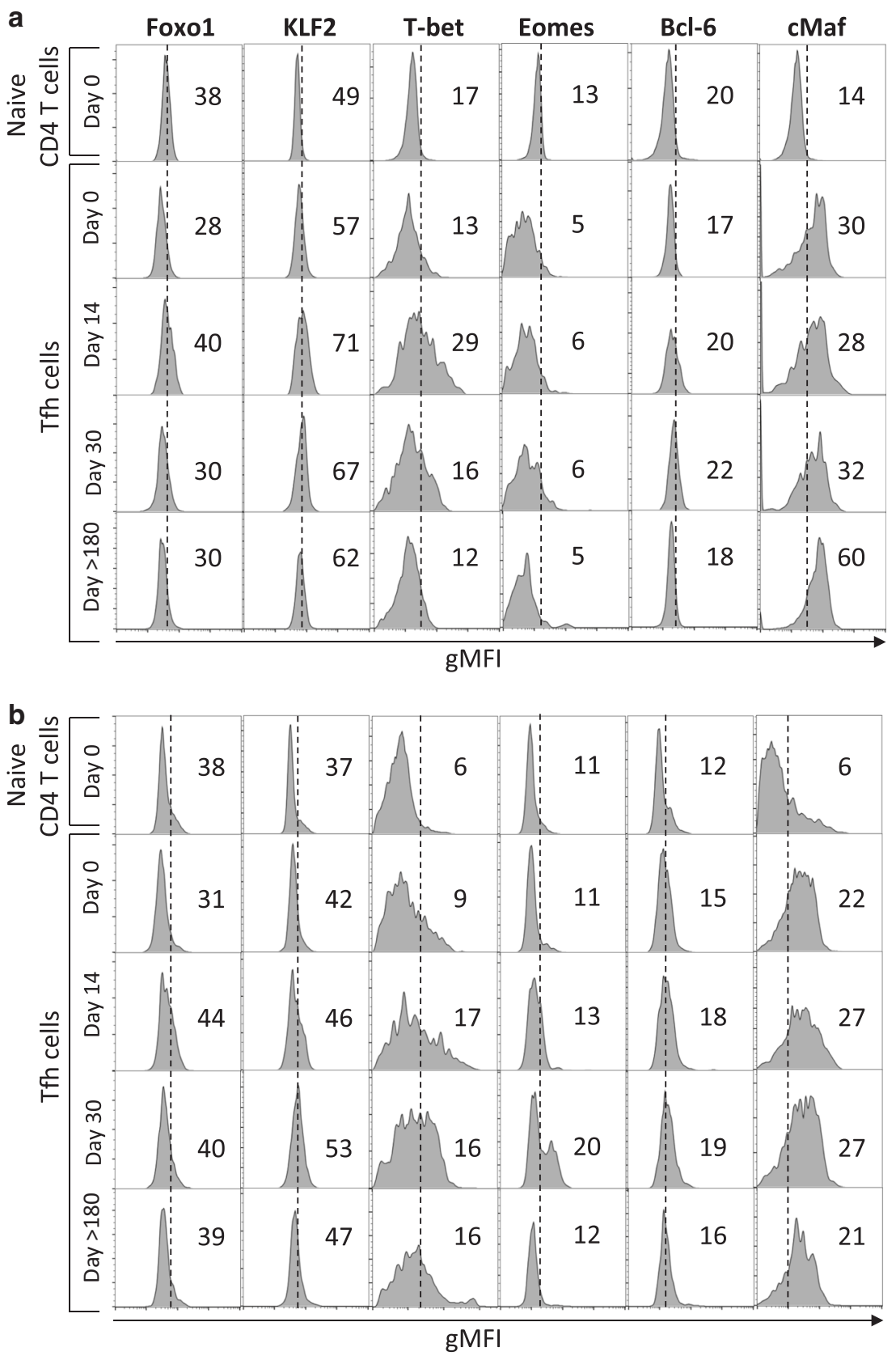

Fig. 7 Expression of transcriptional factors in the Tfh cells of SIV-infected RMs. a, b Histograms show the expression profile of Foxo1, KLF2, Tbet, Eomes, and c-Maf in Tfh cells of a lleocolic and $\mathbf{b}$ colic MLNs from healthy (day 0 ) and SIV-infected RMs at days 14, 30, and >180 compared to naive CD4 T cells of a healthy RM and performed the same day for comparison. The histograms shown are representative of three independent experiments. Mean fluorescence intensities are indicated

impact Tfh function. ${ }^{43,44,49,59,78}$ Importantly, we observed that Tfh cells from SIV-infected RMs display higher levels of Stat5 phosphorylation. Although we did not modulate in vivo IL-27 in SIV-infected RMs due to the actual absence of cross-reacting reagents such as neutralizing antibody, our results performed in vitro and the phenotype associated with Tfh cells derived from MLNs of SIV-infected RMs strongly suggested that in the absence of the IL-12, IL-27 may contribute to the impairment of mucosal Tfh cells during SIV infection.

For the first time, to our knowledge, our results also highlighted that IL-27 modulates the expression of critical TFs, namely KLF2 and Foxo1, which have been reported to inhibit CXCR5 and CD62L expressions. ${ }^{53,54}$ Herein, we found that Foxo1 is increased in the Tfh cells of MLNs, and a switch for an effector memory to a central memory profile (CD45RA ${ }^{-} \mathrm{CD}_{2} \mathrm{~L}^{+}$) is observed. Furthermore, our results indicate that IL-27 inhibits the expression of TCF1 in vitro, which is induced by IL-6/IL-21 and contributes to the differentiation of Tfh cells. ${ }^{58,96}$ We observed that the expression of TCF1 is transiently expressed (day 14) in the Tfh cells of SIV-infected RMs. Therefore, once again for the first time as far as we know, we established the TF networking associated with IL-27 in Tfh cells. Thus, our results indicate that IL-27 in monkeys may contribute to the genesis of Th1-like Tfh cell population by impairing CXCR5 expression and inducing inhibitory TFs that may have an impact on the distribution and function of Tfh cells in GCs during SIV infection. However, we cannot exclude the role of additional cytokines in regulating mucosal Tfh cells in SIV-infected RMs. Indeed, our results indicated that in vitro IL-7 also decreases 
a

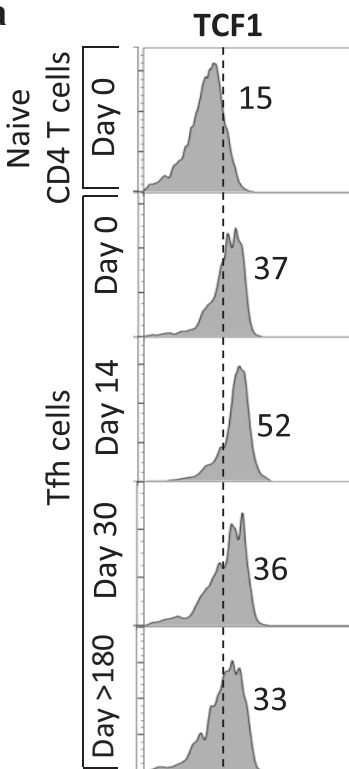

gMFI

C
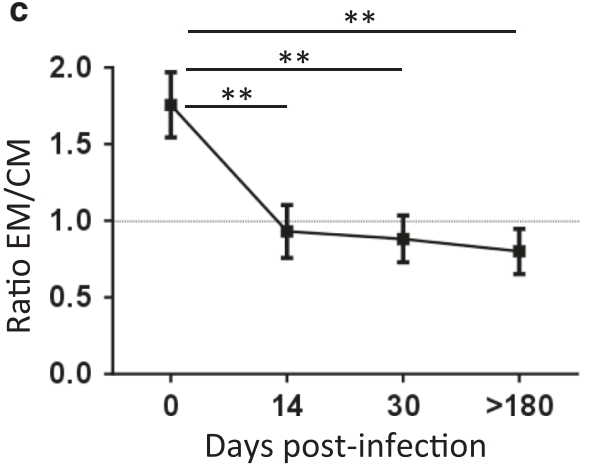

e

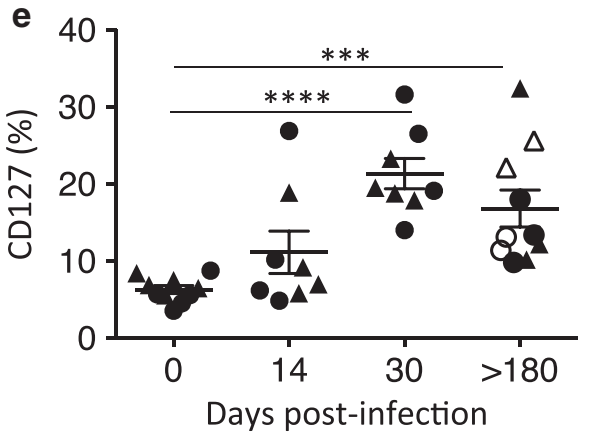

b
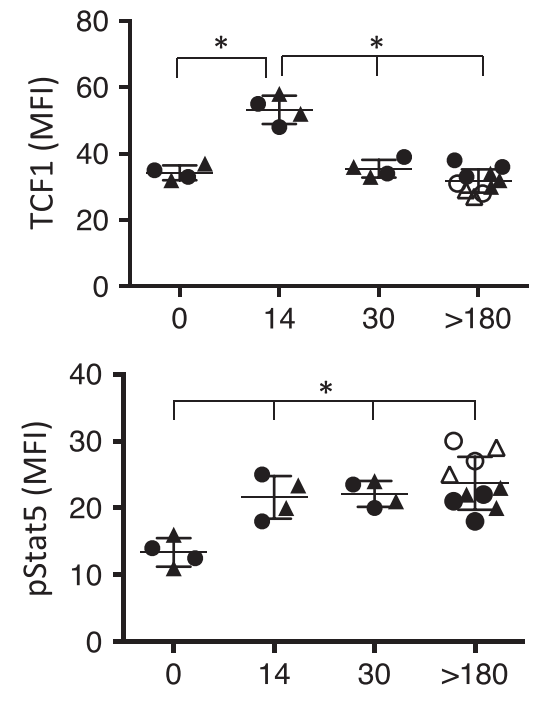

d

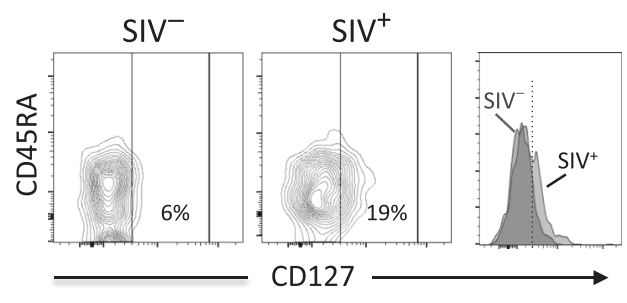

Fig. 8 Expression of TCF1, pStat5, and CD127 in the Tfh cells of SIV-infected RMs. a Expression profile of pStat5 and TCF1 in Tfh cells of colic MLNs from healthy (day 0) and SIV-infected RMs at days 14, 30, and >180 in comparison to naive CD4 T cells from a healthy RM. Mean fluorescence intensities are indicated. b Histograms show the expression of TCF1 and pStat5 in Tfh cells from ileocolic MLNs (triangle) and

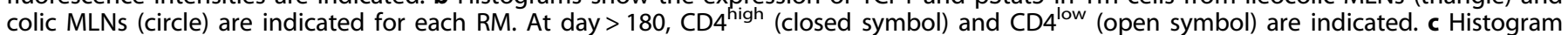
shows the ratio of effector memory (CDR45 ${ }^{-} \mathrm{CD} 62 \mathrm{~L}^{-}$) vs. central memory $\left(\mathrm{CDR} 45^{-} \mathrm{CD} 62 \mathrm{~L}^{+}\right)$Tfh in MLNs of SIV-infected RMs at days $0,14,30$, and $>180$ postinfection. Data are the mean \pm SEM of four individuals at each time point. $\mathbf{d}$ Representative dot plots depicting the expression of CD127 on Tfh cells in uninfected (SIV ${ }^{-}$) and SIV-infected RMs $\left(\mathrm{SIV}^{+}\right)$. Overlay is shown on the right part. d Histograms show the percentage of Tfh cells expressing CD127 at days $0,14,30$, and $>180$ postinfection. Ileocolic MLNs (triangle) and colic MLNs (circle) are indicated for each RM. At day $>180, C D 4^{\text {high }}$ (closed symbol) and $C D 4^{\text {low }}$ (open symbol) are indicated. Statistical analyses were performed using the Mann-Whitney test. ${ }^{*} p<0.05 ;{ }^{* *} p<0.01 ;{ }^{* * *} p<0.001 ;{ }^{* * * *} p<0.0001$

CXCR5 expression. Interestingly, whereas Th cells express low levels of CD127 in healthy RMs, a sustained increase in the expression of CD127 at the cell surface of Tfh cells in MLNs of SIVinfected RMs was observed. Therefore, in a context in which IL-7 has been reported to be increased in the lymphoid tissues and plasma of HIV-1-infected individuals, ${ }^{97-99}$ IL-7 may also contribute to the inhibition of Tfh cell differentiation in the MLNs of SIVinfected RMs. Although we identified a TF network associated with Tfh differentiation during SIV infection, this cannot exclude the role of additional TFs reported to contribute to their regulation 
a

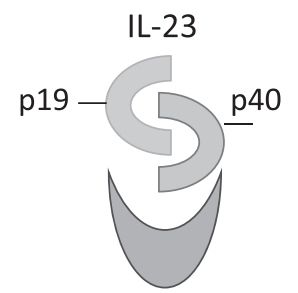

IL-23R
IL-12

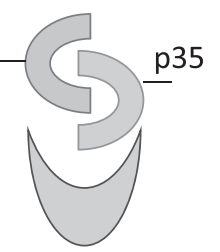

IL-12R
IL-35

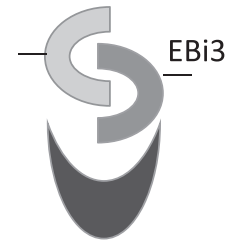

IL-35R
IL-27

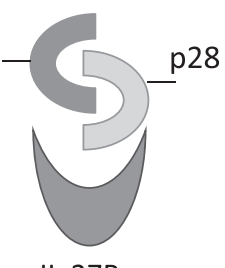

IL-27R
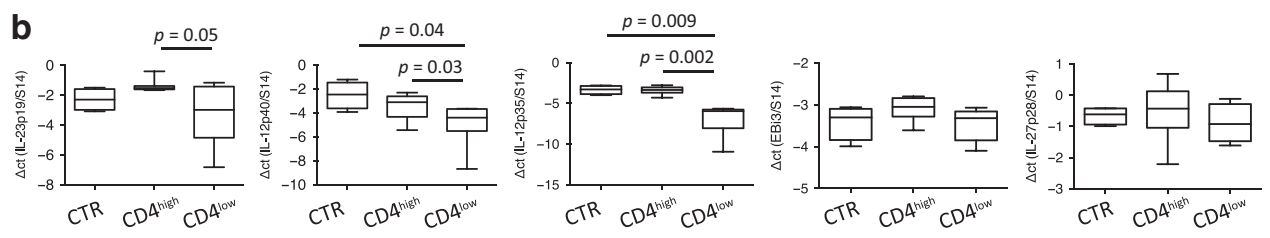

c

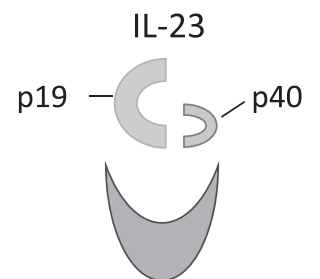

IL-23R

IL-12

IL-35

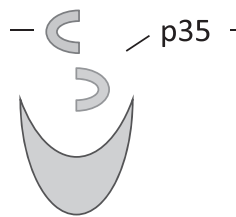

IL-12R

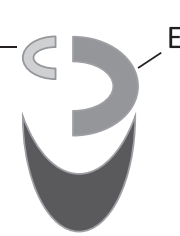

IL-35R
IL-27
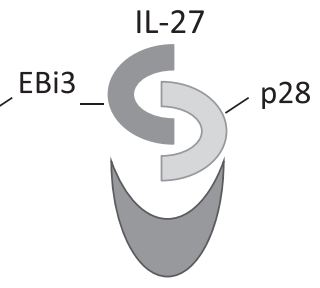

Fig. 9 mRNA expression of the IL-12 cytokine family members in the MLNs of SIV-infected RMs. a Schematic representation of the IL-12 cytokine family, including IL-23, IL-12, IL-35, and IL-27. b mRNA expressions of the $\alpha$ and $\beta$ chains of IL-12 family members in the MLNs of healthy $(n=4), \mathrm{CD}^{\text {high }}(n=4)$ and $\mathrm{CD}^{\text {low }}(n=3)$. Statistical analyses were performed using the Mann-Whitney test. c Schematic representation of the IL-12 cytokine family in MLNs of SIV-infected RMs in which the levels of CD4 is low

such as the transcription factor achaete-scute homolog 2 (Ascl2) as well as the inhibitor of DNA-binding 2 (Id2) and Id $3^{100,101}$ demonstrating the complexity of Th regulation in the context of infectious diseases. Interestingly, several chronic infectious diseases such as malaria and leishmania have also been reported to be associated with the abnormal differentiation and redistribution of Th cells in lymphoid tissues, ${ }^{102,103}$ but the role of IL-27 in regulating Tfh cells has not been addressed in each of these models.

Tfh cells are essential to the regulation of GC size, B-cell differentiation, and B-cell lg affinity maturation. ${ }^{17,18,20}$ Herein, we reported that the percentages of $\mathrm{AM}\left(\mathrm{CD} 21^{-} \mathrm{CD} 27^{+}\right)$and $\mathrm{RM}$ $\left(\mathrm{CD} 21^{+} \mathrm{C} 27^{+}\right) \mathrm{B}$ cells declined in the MLNs of chronically SIVinfected RMs, and a positive correlation between Tfh cells and AM $B$ cells was observed. Furthermore, GC B cells that expressed Fas (CD95) progressively declined. In particular, AM B cells expressing CD95 are lowered in SIV-infected RMs with low level of CD4 T cells compared to RMs having high level of CD4 T cells in MLNs. It is well-known that CD95 contributes to the regulation of memory $B$ cells. ${ }^{75-78}$ The co-engagement of CD40 and the antigen-receptor protect B cells from CD95 ligation-mediated cell death ${ }^{104,105}$ through NF-kB activation. ${ }^{106}$ Therefore, the loss of Tfh cells may lead to the absence of co-stimulatory molecules that are essential for B-cell survival and differentiation leading to the depletion of $B$ cells expressing CD95 in MLNs. Earlier reports have indicated that dying B cells are localized in B-cell areas surrounding GCs where Tfh are normally localized, leading to a polyclonal B-cell activation in intestine and GC involution in HIV-infected individuals, early after infection. ${ }^{107}$ Although we did not directly investigate the specificity of Tfh cells in MLNs, the precocity of Tfh depletion argues against a SIV specificity of these Tfh cells and supported the idea of a more global defect that may have a major impact on the quality of the immune repertoire. In this context, it has been reported that the frequency and quality of Env-specific Tfh cells correlates with the genesis of Env-specific $B$ cells and neutralization and negatively correlates with the expression of Tbet. ${ }^{37}$ An enrichment of abnormal B-cell subsets was reported in viremic patients ${ }^{108}$ that is restored by early antiretroviral therapy ${ }^{109}$ indicating that a defect occurs early after the moment of infection. ${ }^{38-40}$ In the spleen of SIV-infected RMs, we also recently reported an early defect in the differentiation of $B$ cells. ${ }^{33}$ A loss of $B$ cells and an absence of seroconversion was also reported in monkeys, which rapidly progressed to AIDS. ${ }^{110}$ The comparison of pathogenic and nonpathogenic monkeys has also indicated a correlation between the levels of CD4 T cell depletion and the capacity to generate an IgG response. ${ }^{11,72}$ Therefore, the depletion and the profile of Tfh cells derived from SIV-infected RMs may contribute to the general observation that AIDS is associated with the phenotypic and functional abnormalities of $B$ cells that arise over the course of the infection.

Petrovas et al. ${ }^{34}$ have reported that preserved LNs architecture in chronically SIV-infected RMs is associated with increased numbers of Tfh cells. Other groups indicated the loss of Tfh cells in progressor, compared to nonprogressor SIV-infected RMs, ${ }^{36,37}$ and monkeys progressing faster to AIDS exhibit the loss of IL-21secreting cells associated with involution of the GCs. ${ }^{111}$ Herein, we observed lower level of IL-21-secreting cells in MLN follicles, which is associated with a redistribution and higher expression of CXCL13, the ligand of CXCR5. Interestingly, it has been shown that such CXCL13 staining is generally observed during late embryonic splenic vasculature, in which B cells are essentially immature. ${ }^{73}$ This spatial alteration of CXCL13 during SIV infection may contribute to the defect of Tfh cells in MLNs. Indeed, CXCR5 play a critical role in regulating the homing of memory CD4 T cells to the B-cell zones, ${ }^{112,113}$ and Tfh cells are subsequently lost in the absence of B-cell integration. ${ }^{85}$

Because MLNs are not only crucial for the immune response against pathogens, but are also essential to maintain commensal microbiota under immune control, and to maintain immune tolerance, this defect of Th cells reported in MLNs of SIV-infected 
a

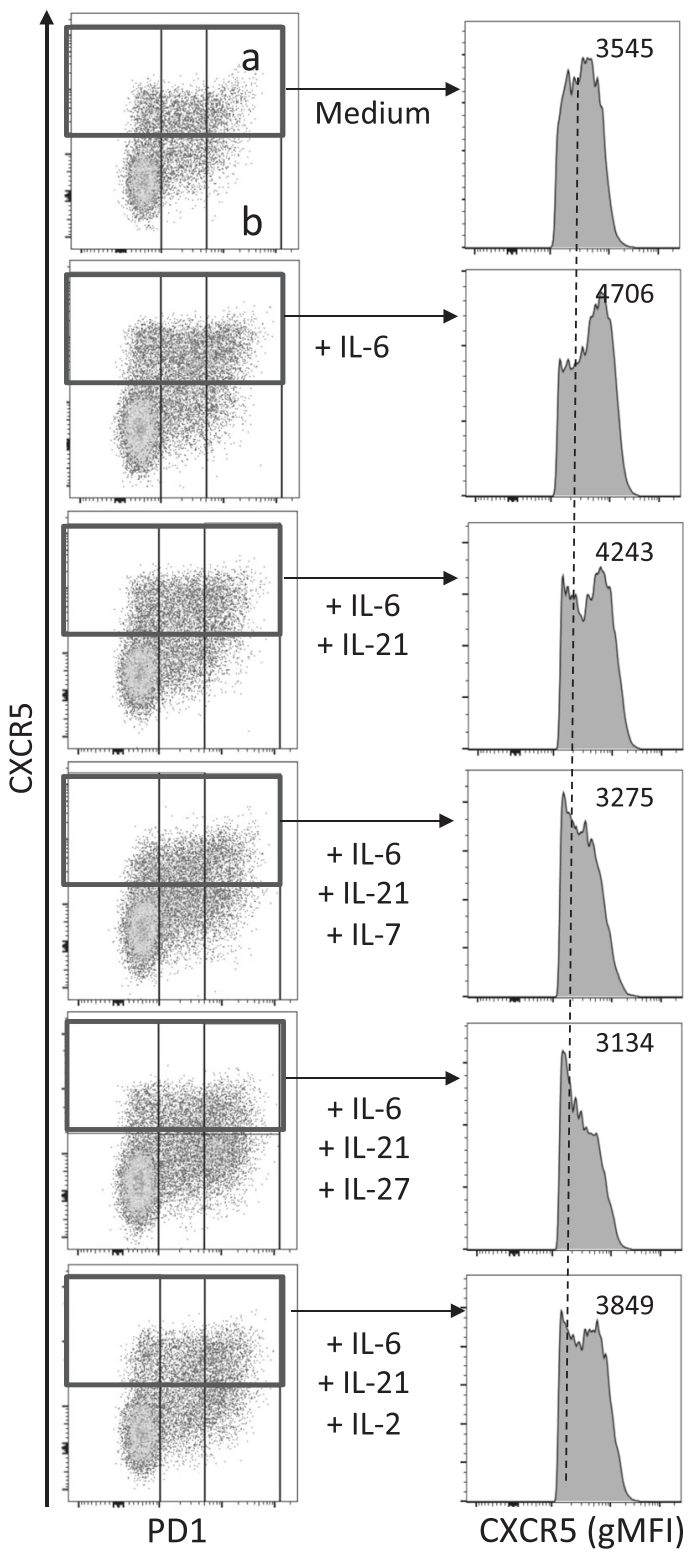

b

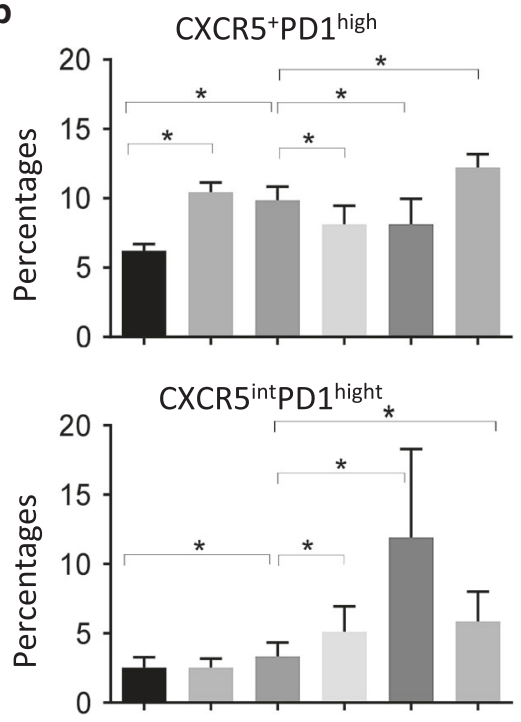

C

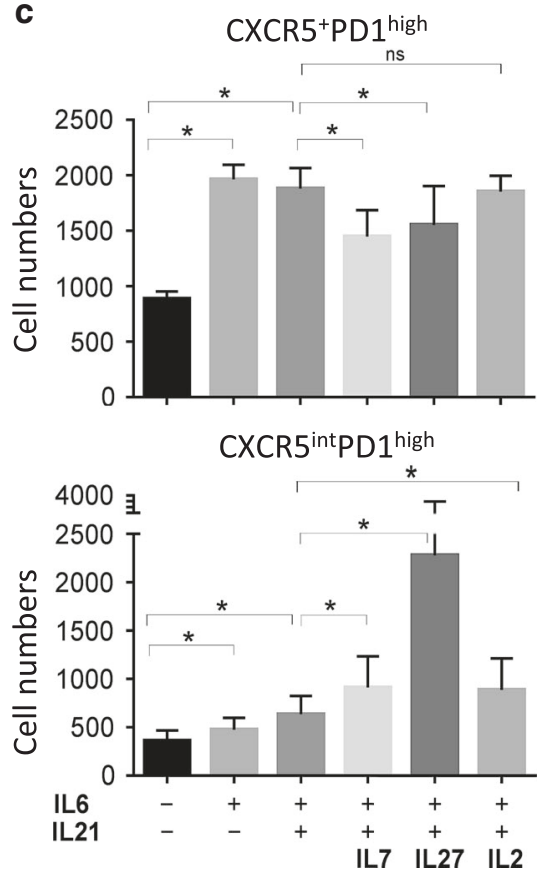

Fig. 10 Impact of IL-27 on Tfh cells derived from MLNs. (a, left panel) Dot plots depicting the expression of CXCR5 and PD1 after stimulation of MLN cell suspension isolated from a healthy RM with coated CD3 and CD28 mAbs ( 0.1 and $0.25 \mu \mathrm{g} / \mathrm{ml}$, respectively) in the absence (medium) or presence of IL-6, IL-21, IL-7, IL-27, and IL-2. (a, right panel) Histograms show the fluorescence intensity of CD4 T cells expressing CXCR5 (gMFI) gating on the region depicted in red. b, c Histograms show the percentages and cell numbers of CXCR5 ${ }^{+}$PD $1^{\text {high }}$ (region a) and CXCR5 ${ }^{\text {low } P D} 1^{\text {high }}$ (region b). Data are the mean \pm SEM of three independent experiments. Statistical analyses were performed using the Mann-Whitney test. ${ }^{*} p<0.05$

RMs may contribute to microbial translocation and inflammation associated with AIDS. These results in monkeys may provide major advances for the understanding of mucosal immunology in humans. Therefore, strategies aiming to preserve Tfh cells can be beneficial to restore B-cell immunity during HIV and SIV infections.

\section{MATERIALS AND METHODS}

Ethics statement

All RMs were housed at Laval University, in accordance with the rules and regulations of the Canadian Council on Animal Care (http://www.ccac.ca). This protocol was approved by the Laval University Animal Protection Committee (Project number 106004).
Animals were fed standard monkey chow diet, supplemented daily with fruit and vegetables, and water ad libitum. Social enrichment was delivered and overseen by a veterinary staff, and overall animal health was monitored daily. Animals showing significant signs of distress, disease, and weight loss were evaluated clinically and were humanely euthanized, using an overdose of barbiturates, according to the guidelines of the Veterinary Medical Association.

Animal, viral inoculation, and sample collection

RMs (Macaca mulatta) seronegative for SIVmac, STLV-1 (Simian T Leukemia Virus type-1), SRV-1 (type D retrovirus) and herpes-B viruses were used in this study. RMs were infected intravenously 
a

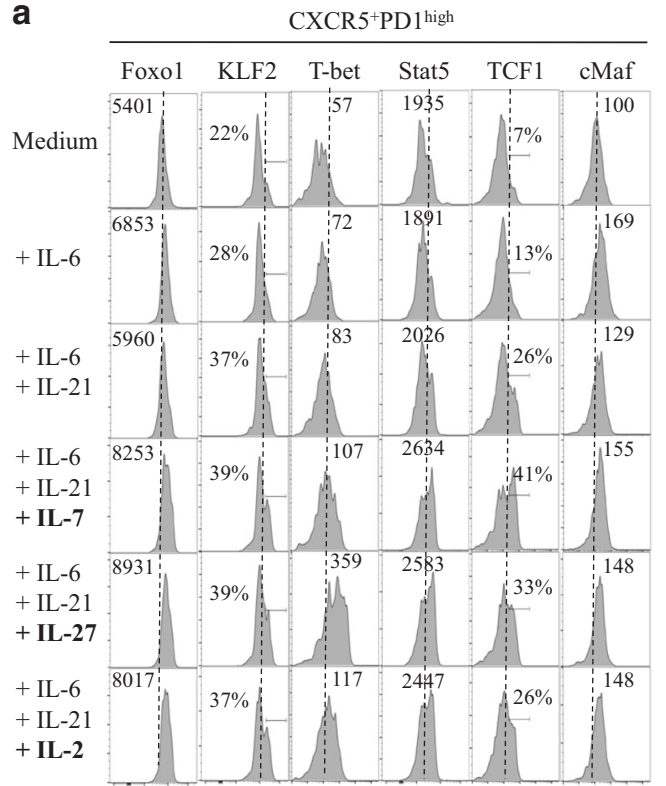

b

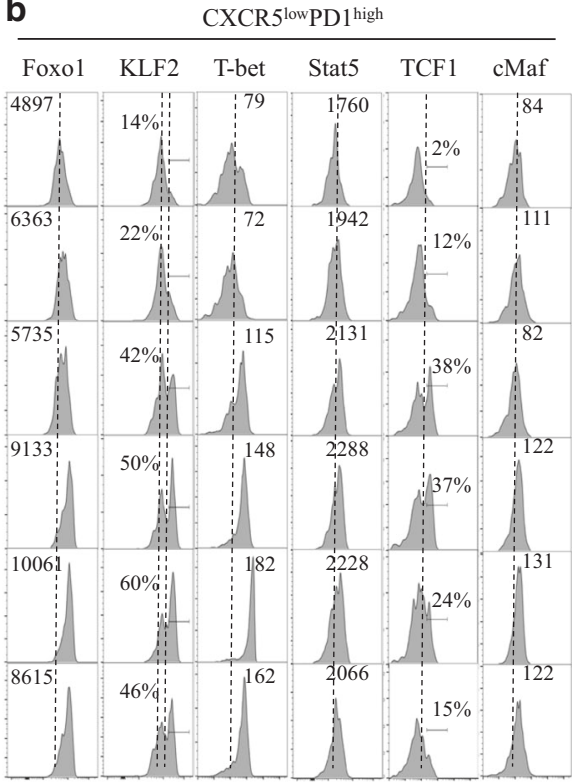

Fig. 11 Impact of IL-27 on the expression of transcriptional factors in Tfh cells. Histograms show the expression profile of Foxo1, KLF2, pStat5, TFC1, and C-Maf in CXCR5 ${ }^{+}$PD $1^{\text {high }}\left(\mathbf{a}\right.$, area a in Fig. 10) and CXCR5 ${ }^{\text {low } P D} 1^{\text {high }}$ (b, area b in Fig. 10) subsets. Thus, data are representative of three independent experiments

with SIVmac251 virus (20 AID 50 ). RMs were euthanized at different time points post-infection, covering both acute and chronic phases. Peripheral blood, spleen, axillary, and inguinal LNs, ileocolic including cisterna chyli, and colic MLNs were recovered for cellular analysis (as shown in supplementary Fig. 4). Cell numbers were calculated from LNs retrieved in each region (the totality of the MLNs were retrieved). For blood, a hemogram was elaborated using an Abaxis VetScan HM5 hematology instrument (Abaxis, CA). Tissues were not digested with collagenase or other proteases for cell isolation limiting side effects on the expression of cell surface markers.

\section{Immunophenotyping}

Fresh cells were stained with a panel of monoclonal antibodies. The fluorochrome-conjugated antibodies used are provided in the Table S1. After lysing erythrocytes (Lysing buffer Pharm Lyse 10x BD Biosciences), 60,000 events corresponding to mononuclear cells were recorded in FACS Canto A (BD Bioscience). Intracellular $\mathrm{Bcl}-6$, c-Maf, TCF1, Foxo1, KLF2, Eomes, and T-bet staining was performed after fixing and permeabilizing the cells with the FoxP3 staining buffer set (eBioscience), whereas pStat5 was detected after permeabilization using BD Cytofix ${ }^{\text {TM }}$ Fixation Buffer. Analyses were performed using FlowJo software (Tree Star, Inc.).

In vitro stimulation

MLN cells $\left(1 \times 10^{6}\right)$ were cultured in 24-well plates pre-coated with purified anti-CD3 $(0.1 \mu \mathrm{g} / \mathrm{ml}$, Abcam) and with CD28 $(0.25 \mu \mathrm{g} / \mathrm{ml}$, Biolegend) in the presence of IL-6 (100 ng), and IL-21 (100 ng), and in the absence or presence of IL-27 (10 ng), IL-2 (10 ng), and IL-7 (10 ng). Four (4) days later, cells were stained with anti-PD1-PerCPeFluor710, anti-CXCR5-PE, anti-CD4-APC-H7 and anti-CD20-PE-Cy7. The expressions of Foxo1, KLF2, STAT5, T-bet-, c-Maf- and TCF1 were assessed by flow cytometry.

Immunofluorescence confocal microscopy of tissue sections MLNs were embedded in optimal cutting temperature compound (OCT), sectioned at a $7.5 \mu \mathrm{m}$ thickness and stored unfixed at $-20^{\circ} \mathrm{C}$ until use. Tissue sections were fixed in 4\% PFA (15 min at room temperature) followed by acetone $\left(20 \mathrm{~min}\right.$ at $\left.-20^{\circ} \mathrm{C}\right)$. Slides were submerged in blocking solution ( $5 \%$ normal horse serum, $0.3 \%$ triton $\mathrm{X}-100)$ for $1 \mathrm{~h}$ at room temperature. For panel 1 (CD4/CD20/ CXCR5/PD1), sections were initially incubated overnight at $4{ }^{\circ} \mathrm{C}$ with a purified anti-CXCR5 antibody (kindly provided by the $\mathrm{NIH}$ Nonhuman Primate Reagent Resource) diluted in antibody dilution buffer ( $1 \%$ bovine serum albumin, $0.3 \%$ triton X-100). The next day, sections were washed and incubated with a secondary antibody coupled with Alexa Fluor-488 (AF-488) diluted in antibody dilution buffer for $1 \mathrm{~h}$ at room temperature. After extensive washing, sections were incubated overnight at $4{ }^{\circ} \mathrm{C}$ with fluorochrome-coupled antibodies, including the CD4-BV421, CD20-eF615 and PD1-AF647. After washing, the slides were mounted with Prolong Gold antifade mounting medium. The procedure employed for Panel 2 (IL-21/ CXCL13/CD20) was similar. Briefly, sections were initially incubated with a purified anti-IL21 antibody and a purified anti-CXCL13 antibody overnight, followed by two secondary antibodies, one coupled to AF-488 and one coupled to AF-546. After washing, samples were incubated overnight with the directly coupled antibody for CD20-eF660. S1 Table provides information regarding the antibodies used for tissue immunofluorescence. Sections were imaged in a Zeiss LSM 710 confocal microscope. Tiled Z-stacks were acquired with a 20x objective and stitched using the Image J stitching plugin. ${ }^{114}$ Average intensity projections were obtained from the stitched tiles using built-in Image $J$ tools.

In situ hybridization

Productively infected cells (SIV ${ }^{+}$RNA cells) were assessed in LNs by in situ hybridization, as previously described. ${ }^{16}$ Infected cells were detected and counted in the paracortical zone on a minimum of three sections using a Nikon-FXA microscope. $A{ }^{35}$ S-labeled RNA probe derived from the SIVmac nef gene was used. To enhance probe penetration into the tissue sections, the ${ }^{35}$ S-labeled RNA was subjected to mild alkaline hydrolysis to obtain a majority of fragments in the 150- to 200-nucleotide range. The number of positive cells was then divided by the surface of the entire LN section, and the results were expressed as the number of positive cells per $2-\mathrm{mm}^{2}$ section. The mean count was calculated for three slides of the same LNs obtained in a blinded fashion by two different investigators. 
Statistical analysis

Statistics were performed with GraphPad Prism 5 software. The nonparametric Mann-Whitney test and Wilcoxon tests were used, as indicated.

\section{ACKNOWLEDGEMENTS}

This work was supported by grants from the Canadian Institutes of Health Research (CIHR) (HBF-123682, HBF-126786, and MOP-133476), the ANRS (France), and by the Canadian HIV Cure Enterprise Grant HIG-133050 from the CIHR partnership with CANFAR and IAS. F.M. and H.R. were supported by the "Fondation du CHU de Québec", and Y.F. by the ANRS. We also thank Daphnée Veilleux-Lemieux and AnneMarie Catudal for their help at the nonhuman primate center (Quebec City). J.E. thanks the Canada Research Chair program for financial assistance.

\section{AUTHOR CONTRIBUTIONS}

F.M., H.R., Y.F., V.R., J.C., G.B.L., O.Z.A. and C.S. performed the experiments, V.R., M.L. and J.E. designed the work, and V.R. and J.E. wrote the paper.

\section{ADDITIONAL INFORMATION}

The online version of this article (https://doi.org/10.1038/s41385-019-0174-0) contains supplementary material, which is available to authorized users.

Competing interests: The authors declare no competing interests.

Publisher's note: Springer Nature remains neutral with regard to jurisdictional claims in published maps and institutional affiliations.

\section{REFERENCES}

1. Deeks, S. G. et al. Immune activation set point during early HIV infection predicts subsequent CD4+ T-cell changes independent of viral load. Blood 104, 942-947 (2004).

2. Estaquier, J. et al. T helper type $1 / T$ helper type 2 cytokines and $\mathrm{T}$ cell death: preventive effect of interleukin 12 on activation-induced and CD95 (FAS/APO1)-mediated apoptosis of CD4+ T cells from human immunodeficiency virusinfected persons. J. Exp. Med. 182, 1759-1767 (1995).

3. Estaquier, J. et al. Fas-mediated apoptosis of CD4+ and CD8+ T cells from human immunodeficiency virus-infected persons: differential in vitro preventive effect of cytokines and protease antagonists. Blood 87, 4959-4966 (1996).

4. Katsikis, P. D. et al. Fas antigen stimulation induces marked apoptosis of T lymphocytes in human immunodeficiency virus-infected individuals. J. Exp. Med. 181, 2029-2036 (1995).

5. Arnoult, D. et al. Caspase-dependent and -independent T-cell death pathways in pathogenic simian immunodeficiency virus infection: relationship to disease progression. Cell Death Differ. 10, 1240-1252 (2003).

6. Estaquier, J. et al. Programmed cell death and AIDS: significance of T-cell apoptosis in pathogenic and nonpathogenic primate lentiviral infections. Proc. Natl Acad. Sci. USA 91, 9431-9435 (1994).

7. Finkel, T. H. et al. Apoptosis occurs predominantly in bystander cells and not in productively infected cells of HIV- and SIV-infected lymph nodes. Nat. Med. 1, 129-134 (1995).

8. Katsikis, P. D. et al. Activation-induced peripheral blood T cell apoptosis is Fas independent in HIV-infected individuals. Int Immunol. 8, 1311-1317 (1996).

9. Li, Q. et al. Peak SIV replication in resting memory CD4+ T cells depletes gut lamina propria CD4+ T cells. Nature 434, 1148-1152 (2005).

10. Mattapallil, J. J. et al. Massive infection and loss of memory CD4+ T cells in multiple tissues during acute SIV infection. Nature 434, 1093-1097 (2005).

11. Monceaux, V. et al. Extensive apoptosis in lymphoid organs during primary SIV infection predicts rapid progression towards AIDS. Aids 17, 1585-1596 (2003).

12. Silvestri, G. et al. Nonpathogenic SIV infection of sooty mangabeys is characterized by limited bystander immunopathology despite chronic high-level viremia. Immunity 18, 441-452 (2003).

13. Brenchley, J. M. et al. CD4+ T cell depletion during all stages of HIV disease occurs predominantly in the gastrointestinal tract. J. Exp. Med. 200, 749-759 (2004).

14. Mehandru, S. et al. Primary HIV-1 infection is associated with preferential depletion of CD4+ T lymphocytes from effector sites in the gastrointestinal tract. J. Exp. Med. 200, 761-770 (2004).

15. Mowat, A. M. Anatomical basis of tolerance and immunity to intestinal antigens. Nat. Rev. Immunol. 3, 331-341 (2003).
16. Cumont, M. C. et al. TGF-beta in intestinal lymphoid organs contributes to the death of armed effector CD8 T cells and is associated with the absence of virus containment in rhesus macaques infected with the simian immunodeficiency virus. Cell Death Differ. 14, 1747-1758 (2007).

17. Crotty, S. Follicular helper CD4 T cells (TFH). Annu Rev. Immunol. 29, 621-663 (2011).

18. Vinuesa, C. G. et al. Follicular B helper T cells in antibody responses and autoimmunity. Nat. Rev. Immunol. 5, 853-865 (2005).

19. Fahey, L. M. et al. Viral persistence redirects CD4 T cell differentiation toward T follicular helper cells. J. Exp. Med. 208, 987-999 (2011).

20. Crotty, S. T follicular helper cell differentiation, function, and roles in disease. Immunity 41, 529-542 (2014).

21. Linterman, M. A. et al. IL-21 acts directly on B cells to regulate $\mathrm{BCl}-6$ expression and germinal center responses. J. Exp. Med. 207, 353-363 (2010).

22. Poholek, A. C. et al. In vivo regulation of $\mathrm{Bcl} 6$ and $\mathrm{T}$ follicular helper cell development. J. Immunol. 185, 313-326 (2010).

23. Zotos, D. et al. IL-21 regulates germinal center B cell differentiation and proliferation through a B cell-intrinsic mechanism. J. Exp. Med. 207, 365-378 (2010).

24. Ansel, K. M. et al. In vivo-activated CD4 T cells upregulate CXC chemokine receptor 5 and reprogram their response to lymphoid chemokines. J. Exp. Med. 190, 1123-1134 (1999).

25. Johnston, R. J. et al. Bcl6 and Blimp-1 are reciprocal and antagonistic regulators of T follicular helper cell differentiation. Science 325, 1006-1010 (2009).

26. Kim, C. H. et al. Subspecialization of CXCR5 + T cells: B helper activity is focused in a germinal center-localized subset of CXCR5+ T cells. J. Exp. Med. 193, 1373-1381 (2001).

27. Gunn, M. D. et al. A B-cell-homing chemokine made in lymphoid follicles activates Burkitt's lymphoma receptor-1. Nature 391, 799-803 (1998).

28. Legler, D. F. et al. B cell-attracting chemokine 1, a human CXC chemokine expressed in lymphoid tissues, selectively attracts B lymphocytes via BLR1/ CXCR5. J. Exp. Med. 187, 655-660 (1998).

29. Boswell, K. L. et al. Loss of circulating CD4 T cells with B cell helper function during chronic HIV infection. PLoS Pathog. 10, e1003853 (2014).

30. Brenchley, J. M. et al. Differential infection patterns of CD4+ T cells and lymphoid tissue viral burden distinguish progressive and nonprogressive lentiviral infections. Blood 120, 4172-4181 (2012).

31. Cubas, R. A. et al. Inadequate $T$ follicular cell help impairs B cell immunity during HIV infection. Nat. Med. 19, 494-499 (2013).

32. Fukazawa, Y. et al. B cell follicle sanctuary permits persistent productive simian immunodeficiency virus infection in elite controllers. Nat. Med. 21, 132-139 (2015).

33. Moukambi, F. et al. Early loss of splenic Tfh cells in SIV-infected rhesus macaques. PLoS Pathog. 11, e1005287 (2015).

34. Petrovas, C. et al. CD4 T follicular helper cell dynamics during SIV infection. J. Clin. Invest. 122, 3281-3294 (2012).

35. lannello, A. et al. Dynamics and consequences of IL-21 production in HIVinfected individuals: a longitudinal and cross-sectional study. J. Immunol. 184, 114-126 (2010).

36. $\mathrm{Xu}, \mathrm{H}$. et al. Persistent simian immunodeficiency virus infection causes ultimate depletion of follicular Th cells in AIDS. J. Immunol. 195, 4351-4357 (2015).

37. Yamamoto, T. et al. Quality and quantity of TFH cells are critical for broad antibody development in SHIVAD8 infection. Sci. Transl. Med. 7, 298ra120 (2015).

38. Morris, L. et al. HIV-1 antigen-specific and -nonspecific B cell responses are sensitive to combination antiretroviral therapy. J. Exp. Med. 188, 233-245 (1998).

39. Titanji, K. et al. Primary HIV-1 infection sets the stage for important B lymphocyte dysfunctions. Aids 19, 1947-1955 (2005).

40. Titanji, K. et al. Loss of memory B cells impairs maintenance of long-term serologic memory during HIV-1 infection. Blood 108, 1580-1587 (2006).

41. Schmitz, J. E. et al. Inhibition of adaptive immune responses leads to a fatal clinical outcome in SIV-infected pigtailed macaques but not vervet African green monkeys. PLoS Pathog. 5, e1000691 (2009).

42. Ivanov, I. I. et al. The orphan nuclear receptor RORgammat directs the differentiation program of proinflammatory IL-17+ T helper cells. Cell 126, 1121-1133 (2006).

43. Johnston, R. J. et al. STAT5 is a potent negative regulator of TFH cell differentiation. J. Exp. Med. 209, 243-250 (2012).

44. Nurieva, R. I. et al. STAT5 protein negatively regulates T follicular helper (Tfh) cell generation and function. J. Biol. Chem. 287, 11234-11239 (2012).

45. Szabo, S. J. et al. A novel transcription factor, T-bet, directs Th1 lineage commitment. Cell 100, 655-669 (2000).

46. Zheng, W. et al. The transcription factor GATA-3 is necessary and sufficient for Th2 cytokine gene expression in CD4 T cells. Cell 89, 587-596 (1997). 
47. Nurieva, R. I. et al. Bcl6 mediates the development of $\mathrm{T}$ follicular helper cells. Science 325, 1001-1005 (2009).

48. $\mathrm{Yu}, \mathrm{D}$. et al. The transcriptional repressor Bcl-6 directs $\mathrm{T}$ follicular helper cell lineage commitment. Immunity 31, 457-468 (2009).

49. Ballesteros-Tato, A. et al. Interleukin-2 inhibits germinal center formation by limiting T follicular helper cell differentiation. Immunity 36, 847-856 (2012).

50. Bauquet, A. T. et al. The costimulatory molecule ICOS regulates the expression of C-Maf and IL-21 in the development of follicular T helper cells and TH-17 cells. Nat. Immunol. 10, 167-175 (2009).

51. Kroenke, M. A. et al. Bcl6 and Maf cooperate to instruct human follicular helper CD4 T cell differentiation. J. Immunol. 188, 3734-3744 (2012).

52. Oestreich, K. J. et al. Molecular mechanisms that control the expression and activity of Bcl-6 in $\mathrm{TH} 1$ cells to regulate flexibility with a TFH-like gene profile. Nat. Immunol. 13, 405-411 (2012).

53. Lee, J. Y. et al. The transcription factor KLF2 restrains CD4(+) T follicular helper cell differentiation. Immunity 42, 252-264 (2015).

54. Weber, J. P. et al. ICOS maintains the T follicular helper cell phenotype by downregulating Kruppel-like factor 2. J. Exp. Med. 212, 217-233 (2015).

55. Fabre, S. et al. FOXO1 regulates L-Selectin and a network of human T cell homing molecules downstream of phosphatidylinositol 3-kinase. J. Immunol. 181, 2980-2989 (2008).

56. Kerdiles, Y. M. et al. Foxo1 links homing and survival of naive $T$ cells by regulating L-selectin, CCR7 and interleukin 7 receptor. Nat. Immunol. 10, 176-184 (2009).

57. $\mathrm{Wu}, \mathrm{T}$. et al. TCF1 is required for the $\mathrm{T}$ follicular helper cell response to viral infection. Cell Rep. 12, 2099-2110 (2015).

58. $\mathrm{Xu}, \mathrm{L}$. et al. The transcription factor TCF-1 initiates the differentiation of $\mathrm{T}(\mathrm{FH})$ cells during acute viral infection. Nat. Immunol. 16, 991-999 (2015).

59. McDonald, P. W. et al. IL-7 signalling represses $\mathrm{BCl}-6$ and the TFH gene program. Nat. Commun. 7, 10285 (2016).

60. Ma, C. S. et al. Early commitment of naive human CD4(+) T cells to the $T$ follicular helper $(\mathrm{T}(\mathrm{FH})$ ) cell lineage is induced by IL-12. Immunol. Cell Biol. 87, 590-600 (2009).

61. Schmitt, N. et al. Human dendritic cells induce the differentiation of interleukin21-producing $\mathrm{T}$ follicular helper-like cells through interleukin-12. Immunity 31, 158-169 (2009).

62. Schmitt, N. et al. IL-12 receptor beta1 deficiency alters in vivo T follicular helper cell response in humans. Blood 121, 3375-3385 (2013).

63. Trinchieri, G. et al. The IL-12 family of heterodimeric cytokines: new players in the regulation of T cell responses. Immunity 19, 641-644 (2003).

64. Schmitt, N. et al. The cytokine TGF-beta co-opts signaling via STAT3-STAT4 to promote the differentiation of human TFH cells. Nat. Immunol. 15, 856-865 (2014).

65. Batten, M. et al. IL-27 supports germinal center function by enhancing IL-21 production and the function of $\mathrm{T}$ follicular helper cells. J. Exp. Med. 207, 2895-2906 (2010).

66. Harker, J. A. et al. Cell-intrinsic IL-27 and gp130 cytokine receptor signaling regulates virus-specific CD4(+) T cell responses and viral control during chronic infection. Immunity 39, 548-559 (2013).

67. Gringhuis, S. I. et al. Fucose-based PAMPs prime dendritic cells for follicular T helper cell polarization via DC-SIGN-dependent IL-27 production. Nat. Commun. 5, 5074 (2014).

68. Ruiz-Riol, M. et al. Identification of interleukin-27 (IL-27)/IL-27 receptor subunit alpha as a critical immune axis for in vivo HIV control. J. Virol. 91, pii: e00441-17 (2017).

69. Fakruddin, J. M. et al. Noninfectious papilloma virus-like particles inhibit HIV-1 replication: implications for immune control of HIV-1 infection by IL-27. Blood 109, 1841-1849 (2007).

70. Greenwell-Wild, T. et al. Interleukin-27 inhibition of HIV-1 involves an intermediate induction of type I interferon. Blood 114, 1864-1874 (2009).

71. Chehimi, J. et al. Impaired interleukin 12 production in human immunodeficiency virus-infected patients. J. Exp. Med. 179, 1361-1366 (1994).

72. Cumont, M. C. et al. Early divergence in lymphoid tissue apoptosis between pathogenic and nonpathogenic simian immunodeficiency virus infections of nonhuman primates. J. Virol. 82, 1175-1184 (2008).

73. Neely, H. R. et al. CXCL13 responsiveness but not CXCR5 expression by late transitional B cells initiates splenic white pulp formation. J. Immunol. 194, 2616-2623 (2015).

74. Hong, J. J. et al. Spatial alterations between CD4(+) T follicular helper, B, and CD8(+) T cells during simian immunodeficiency virus infection: T/B cell homeostasis, activation, and potential mechanism for viral escape. J. Immunol. 188, 3247-3256 (2012)

75. Martinez-Valdez, H. et al. Human germinal center B cells express the apoptosisinducing genes Fas, c-myc, P53, and Bax but not the survival gene bcl-2. J. Exp. Med. 183, 971-977 (1996).
76. Hao, Z. et al. Fas receptor expression in germinal-center $B$ cells is essential for $T$ and B lymphocyte homeostasis. Immunity 29, 615-627 (2008).

77. Hennino, A. et al. FLICE-inhibitory protein is a key regulator of germinal center $B$ cell apoptosis. J. Exp. Med. 193, 447-458 (2001).

78. Peter, M. E. et al. The CD95 receptor: apoptosis revisited. Cell 129, 447-450 (2007).

79. Yoshida, $\mathrm{H}$. et al. Regulation of immune responses by interleukin-27. Immunol. Rev. 226, 234-247 (2008).

80. Chougnet, $C$. et al. Molecular analysis of decreased interleukin-12 production in persons infected with human immunodeficiency virus. J. Infect. Dis. 174, 46-53 (1996).

81. Choi, Y. S. et al. Cutting edge: STAT1 is required for IL-6-mediated Bcl6 induction for early follicular helper cell differentiation. J. Immunol. 190, 3049-3053 (2013).

82. Harker, J. A. et al. Late interleukin- 6 escalates T follicular helper cell responses and controls a chronic viral infection. Science 334, 825-829 (2011).

83. Nurieva, R. I. et al. Generation of $T$ follicular helper cells is mediated by interleukin-21 but independent of T helper 1, 2, or 17 cell lineages. Immunity 29, 138-149 (2008).

84. Pflanz, S. et al. WSX-1 and glycoprotein 130 constitute a signal-transducing receptor for IL-27. J. Immunol. 172, 2225-2231 (2004).

85. Choi, Y. S. et al. ICOS receptor instructs T follicular helper cell versus effector cell differentiation via induction of the transcriptional repressor Bcl6. Immunity 34, 932-946 (2011).

86. Iweala, O. I. et al. Immune privilege in the gut: the establishment and maintenance of non-responsiveness to dietary antigens and commensal flora. Immunol. Rev. 213, 82-100 (2006).

87. Macpherson, A. J. et al. Mesenteric lymph nodes at the center of immune anatomy. J. Exp. Med. 203, 497-500 (2006).

88. Worbs, T. et al. Oral tolerance originates in the intestinal immune system and relies on antigen carriage by dendritic cells. J. Exp. Med. 203, 519-527 (2006).

89. Bishop, P. E. et al. Immunological study of the rectal mucosa of men with and without human immunodeficiency virus infection. Gut 28, 1619-1624 (1987).

90. Mattapallil, J. J. et al. Intestinal intraepithelial lymphocytes are primed for gamma interferon and MIP-1beta expression and display antiviral cytotoxic activity despite severe CD4(+) T-cell depletion in primary simian immunodeficiency virus infection. J. Virol. 72, 6421-6429 (1998).

91. Schneider, $T$. et al. Loss of CD4 T lymphocytes in patients infected with human immunodeficiency virus type 1 is more pronounced in the duodenal mucosa than in the peripheral blood. Berlin Diarrhea/Wasting Syndrome Study Group. Gut 37, 524-529 (1995).

92. Veazey, R. S. et al. Gastrointestinal tract as a major site of CD4+ T cell depletion and viral replication in SIV infection. Science 280, 427-431 (1998).

93. $\mathrm{Wu}, \mathrm{Y}$. et al. IL-6 produced by immune complex-activated follicular dendritic cells promotes germinal center reactions, IgG responses and somatic hypermutation. Int. Immunol. 21, 745-756 (2009).

94. Takeda, A. et al. Cutting edge: role of IL-27/WSX-1 signaling for induction of Tbet through activation of STAT1 during initial Th1 commitment. J. Immunol. 170, 4886-4890 (2003).

95. Kamiya, S. et al. An indispensable role for STAT1 in IL-27-induced T-bet expression but not proliferation of naive CD4+ T cells. J. Immunol. 173, 3871-3877 (2004).

96. Choi, Y. S. et al. LEF-1 and TCF-1 orchestrate T(FH) differentiation by regulating differentiation circuits upstream of the transcriptional repressor Bcl6. Nat. Immunol. 16, 980-990 (2015).

97. Boulassel, M. R. et al. Circulating levels of IL-7 but not IL-15, IGF-1, and TGF-beta are elevated during primary HIV-1 infection. HIV Clin. Trials 5, 357-359 (2004).

98. Malaspina, A. et al. Appearance of immature/transitional B cells in HIV-infected individuals with advanced disease: correlation with increased IL-7. Proc. Natl Acad. Sci. USA 103, 2262-2267 (2006).

99. Napolitano, L. A. et al. Increased production of IL-7 accompanies HIV-1-mediated T-cell depletion: implications for T-cell homeostasis. Nat. Med. 7, 73-79 (2001).

100. Liu, X. et al. Transcription factor achaete-scute homologue 2 initiates follicular Thelper-cell development. Nature 507, 513-518 (2014).

101. Miyazaki, M. et al. The opposing roles of the transcription factor E2A and its antagonist Id 3 that orchestrate and enforce the naive fate of T cells. Nat. Immunol. 12, 992-1001 (2011).

102. Obeng-Adjei, N. et al. Circulating Th1-cell-type Tfh cells that exhibit impaired B cell help are preferentially activated during acute malaria in children. Cell Rep. 13, 425-439 (2015).

103. Rodrigues, V. et al. Abortive T follicular helper development is associated with a defective humoral response in Leishmania infantum-infected macaques. PLoS Pathog. 10, e1004096 (2014). 
104. Lagresle, C. et al. Concurrent engagement of CD40 and the antigen receptor protects naive and memory human B cells from APO-1/Fas-mediated apoptosis. J. Exp. Med. 183, 1377-1388 (1996).

105. Rothstein, T. L. et al. Protection against Fas-dependent Th1-mediated apoptosis by antigen receptor engagement in B cells. Nature 374, 163-165 (1995).

106. Luo, W. et al. B cell receptor and CD40 signaling are rewired for synergistic induction of the c-Myc transcription factor in germinal center B cells. Immunity 48, 313-326.e315 (2018).

107. Levesque, M. C. et al. Polyclonal B cell differentiation and loss of gastrointestinal tract germinal centers in the earliest stages of HIV-1 infection. PLoS Med. 6, e1000107 (2009).

108. Moir, S. et al. Decreased survival of B cells of HIV-viremic patients mediated by altered expression of receptors of the TNF superfamily. J. Exp. Med. 200, 587-599 (2004).

109. Moir, S. et al. B cells in early and chronic HIV infection: evidence for preservation of immune function associated with early initiation of antiretroviral therapy. Blood 116, 5571-5579 (2010).

110. Dykhuizen, M. et al. Determinants of disease in the simian immunodeficiency virus-infected rhesus macaque: characterizing animals with low antibody responses and rapid progression. J. Gen. Virol. 79(Pt 10), 2461-2467 (1998).

111. Hong, J. J. et al. Early lymphoid responses and germinal center formation correlate with lower viral load set points and better prognosis of simian immunodeficiency virus infection. J. Immunol. 193, 797-806 (2014).
112. Breitfeld, D. et al. Follicular B helper T cells express CXC chemokine receptor 5 , localize to $\mathrm{B}$ cell follicles, and support immunoglobulin production. J. Exp. Med. 192, 1545-1552 (2000).

113. Schaerli, P. et al. CXC chemokine receptor 5 expression defines follicular homing T cells with B cell helper function. J. Exp. Med. 192, 1553-1562 (2000).

114. Preibisch, S. et al. Globally optimal stitching of tiled 3D microscopic image acquisitions. Bioinformatics 25, 1463-1465 (2009).

Open Access This article is licensed under a Creative Commons Attribution 4.0 International License, which permits use, sharing, adaptation, distribution and reproduction in any medium or format, as long as you give appropriate credit to the original author(s) and the source, provide a link to the Creative Commons license, and indicate if changes were made. The images or other third party material in this article are included in the article's Creative Commons license, unless indicated otherwise in a credit line to the material. If material is not included in the article's Creative Commons license and your intended use is not permitted by statutory regulation or exceeds the permitted use, you will need to obtain permission directly from the copyright holder. To view a copy of this license, visit http://creativecommons. org/licenses/by/4.0/.

(c) The Author(s) 2019 